\title{
Intracellular Analysis in vivo of Different Barosensitive Bulbospinal Neurons in the Rat Rostral Ventrolateral Medulla
}

\author{
Antonio R. Granata and Stephen T. Kitai \\ The University of Tennessee at Memphis, The Health Science Center, Department of Anatomy and Neurobiology, \\ Memphis, Tennessee 38163
}

Neurons located in the rostral ventrolateral medulla (RVLM) with projections to the intermediolateral column (IML) in the spinal cord were electrophysiologically characterized and anatomically identified using an intracellular recording technique in vivo. A group of spontaneously active neurons was antidromically activated by electrical stimulation of the IML in the thoracic spinal cord $\left(T_{2}-T_{3}\right.$ level). The axonal conduction velocities ranged from $1.5 \mathrm{~m} / \mathrm{sec}$ to $11.0 \mathrm{~m} / \mathrm{sec}$; mean value, $5.5 \pm 2.6 \mathrm{~m} / \mathrm{sec}( \pm \mathrm{SD})$. The firing pattern and changes in membrane potential in relation to the cardiac cycle were investigated in these bulbospinal neurons. A first group discharged action potentials with higher frequency at the end of the diastolic/beginning of the systolic period. The average of the neuronal membrane potentials demonstrated depolarizing potentials at the end of the diastolic/beginning of the systolic period. These depolarizing potentials increased in magnitude when the neurons were hyperpolarized. Therefore, they were characterized as EPSPs. The baroreceptor reflex activation produced by the increase in systemic arterial pressure following intravenous injection of phenylephrine elicited hyperpolarization, a decrease in the rate of discharge, and an increase in the membrane input resistance, suggesting that a disfacilitatory effect was produced by the activation of baroreceptor inputs on these bulbospinal neurons. Conversely, the inactivation of the baroreceptor reflex by intravenous injection of sodium nitroprusside produced depolarization and an increase in the firing rate. These neurons were characterized as baroreceptor-sensitive type I neurons.

A second group of bulbospinal neuron in the RVLM was differentiated from the first group because it demonstrated a decrease in the frequency of discharge at the end of the diastolic/beginning of the systolic period. The average of the membrane potentials showed hyperpolarizing potentials that decreased in magnitude when the neuron was hyperpolarized. These hyperpolarizing potentials occurred at the end of the diastolic/beginning of the systolic period and were reversed in polarity after intracellular injections of chloride ions for several minutes. Therefore, these potentials were

\footnotetext{
Received Jan. 23, 1991; revised July 22, 1991; accepted July 24, 1991

This work was supported by Grants-in-Aid 861294 and 891211 from the American Heart Association to A.R.G. and NIH Grants NS 20702 and NS 23886 to S.T.K. We thank Drs. T. H. Joh and D. H. Park for a generous supply of PNMT and $T H$ antibodies.

Correspondence should be addressed to Antonio R. Granata, M.D., The University of Tennessee at Memphis, College of Medicine, 874 Union Avenue, Memphis, TN 38163.

Copyright (C) 1992 Society for Neuroscience $0270-6474 / 92 / 120001-20 \$ 05.00 / 0$
}

characterized as chloride-dependent IPSPs locked to the cardiac cycle. In some of these neurons, the electrical stimulation of the IML produced, in addition to the antidromic action potential, a monosynaptic EPSP with a shorter latency. Based on these unique characteristics, these neurons were defined as barosensitive type II neurons. During constant baroreceptor inactivation achieved by the hypotension produced by intravenous infusions of sodium nitroprusside, the pattern of discharge of barosensitive type II neurons became very regular, and the IPSPs locked to the cardiac cycle were absent.

The recorded neurons were intracellularly injected with biocytin, incubated with the avidin-Texas red complex, and visualized under fluorescent microscopy. The histological sections were then processed for phenylethanolamine- $N$ methyltransferase (PNMT) immunoreactivity, using a double labeling immunohistofluorescence procedure. Although all neurons were found in the RVLM and 22 out of 25 of them were surrounded by catecholaminergic neurons, none of the investigated barosensitive neurons demonstrated PNMT immunoreactivity. These data provide direct evidence for two different types of sympathoexcitatory bulbospinal neurons in the RVLM, each receiving functionally opposing baroreceptor inputs. Even though they were found close to the PNMT-containing neurons, they did not appear to be catecholaminergic. The results support the idea that noncatecholaminergic bulbospinal neurons in the RVLM are important in cardiovascular control.

It is well known that a group of neurons in the rostral ventrolateral medulla (RVLM) plays a critical role in maintaining normal values of arterial pressure and heart rate by providing tonic excitatory inputs to sympathetic preganglionic neurons in the intermediolateral nucleus (IML) of the thoracic-lumbar spinal cord (Dampney and Moon, 1980; Ross et al., 1984; Granata et al., 1985). Moreover, these neurons are thought of as the final bulbospinal pathway of the baroreceptor reflex arc, which controls the sympathetic vasomotor activity (McAllen et al., 1982; Granata et al., 1983, 1985; Sun and Guyenet, 1985; Terui et al., 1986; Morrison et al., 1988). Changes in cardiovascular parameters elicited by the stimulation of other areas in the brain, as for instance the defense area in the hypothalamus (Hilton et al., 1983) and the fastigial pressor areas (McAllen, 1985), as well as by the stimulation of the somatosympathetic reflex (McAllen, 1985; Stornetta et al., 1989) or the cardiovascular responses to cerebral ischemia, are abolished by lesions of the RVLM. These results are consistent with the idea that neurons in the area provide a common descending pathway for such 
different inputs. Anatomical studies have demonstrated that neurons in the RVLM project to the IML in the thoracic and lumbar spinal cord (Amendt et al., 1979; Ross et al., 1984). Evidence that at least $50 \%$ of these bulbospinal neurons in the RVLM are adrenaline synthesizing was provided by studies combining phenylethanolamine- $N$-methyltransferase (PNMT) immunohistochemistry with retrograde transport of colored fluorescent dyes (Tucker et al., 1987). Furthermore, ultrastructural data have shown that PNMT-containing terminals in the IML, likely originated from these adrenergic neurons in the RVLM (the $\mathrm{Cl}$ group of Hökfelt et al., 1974), make monosynaptic contacts with sympathetic preganglionic neurons in the IML (Milner et al., 1988).

The RVLM receives projections from the nucleus tractus solitarius (NTS) at the level of the terminal fields of baro- and cardiopulmonary receptor afferents (Loewy and Burton, 1978; Ross et al., 1985; Ruggiero and Reis, 1988). In addition, neurons in the (1) area postrema, (2) caudal ventrolateral medulla (A1 area), (3) several hypothalamic subnuclei, (4) nucleus parabrachialis, and (5) the central gray matter send projections to the RVLM (Lovick, 1985, 1988; Ross et al., 1985; Sun and Guyenet, 1986).

The RVLM is a region extending to the ventral surface of the medulla; the dorsal aspect involves the nucleus ambiguus and the nucleus retrofacial, while medially this area is limited by the nucleus gigantocellularis ventralis, the inferior olive, and a group of neurons containing 5-HT and substance $\mathrm{P}$ described by Loewy and McKellar (1981). The lateral limit of the RVLM is the spinal trigeminal nucleus and spinal trigeminal tract, whereas the rostral extension of the RVLM is limited by the caudal border of the facial nucleus. Caudally, the RVLM limit is the rostral tip of the lateral reticular nucleus.

The importance of the RVLM in vasomotor control is supported by the fact that electrolytic or chemical lesion of this area, which specifically inhibits neuronal activity, results in the abolition of the sympathetic vasomotor tone; conversely, specific neuronal depolarization by local application of excitatory amino acids elicits an increase in sympathetic activity and arterial blood pressure (Ross et al., 1984; Granata et al., 1985, 1986).

Electrophysiological studies have identified in different species neurons in the RVLM that are antidromically activated from the spinal cord (Brown and Guyenet, 1984; Barman and Gebber, 1985; Terui et al., 1986). The frequency of discharge of these bulbospinal neurons was modulated by baroreceptor inputs; as a result, the probability of discharge of these neurons was demonstrated to be synchronized to the cardiac cycle (Morrison et al., 1988). In addition, the neuronal activity was inhibited either by electrical stimulation of buffer nerves or by natural stimulation of arterial baroreceptors (Terui et al., 1986). However, these studies were performed using extracellular recording techniques, which are limited to such an extent that the identity of the recorded neurons can only be partially determined and the nature of the inputs received by these neurons not very well understood. These technical limitations account in part for the delay in understanding the precise microcircuitry responsible for generating the tonic sympathetic activity in the RVLM, as well as for the inability to identify the neurotransmitter phenotype of these bulbospinal neurons.

Based on indirect evidence, some studies proposed that the sympathetic excitatory neurons in the RVLM correspond to the PNMT-containing neurons of the C1 group (Ross et al., 1984;
Granata et al., 1985). In support of this idea, it has been demonstrated that the lowest threshold for hypertensive responses evoked in the RVLM, by either electrical or chemical stimulation, overlaps the location of $\mathrm{C} 1$ neurons or their axonal trajectories (Ross et al., 1984; Granata et al., 1985). Furthermore, the projections on the ventral medullary surface of the $\mathrm{Cl}$ area correspond to the chemosensitive zone from which different pharmacological agents modify arterial pressure and heart rate (Benarroch et al., 1986). On the other hand, it is difficult to conciliate with this hypothesis the fact that adrenaline microiontophoretically applied on sympathetic preganglionic neurons in vivo produced depressant effects (Coote et al., 1981; Coote, 1988), while in in vitro preparations, catecholamines produced both depolarization and hyperpolarization (Yoshimura et al., 1989).

The present study was performed to identify directly the bulbospinal neurons in the RVLM, by using an intracellular recording technique in vivo. The recorded neurons were labeled by intracellular injections of biocytin; this technique is combined with immunocytochemical identification of PNMT to determine whether the recorded and electrophysiologically characterized neurons correspond to the $\mathrm{Cl}$ adrenergic group. We have also defined with precision the nature of the synaptic inputs received from baroreceptors by at least two different populations of bulbospinal neurons in the RVLM. The present report represents the first systematic and detailed study of the sympathoexcitatory bulbospinal neurons in the RVLM, using an intracellular recording technique in vivo combined with intracellular labeling and immunostaining against catecholaminergic biosynthetic enzymes.

Preliminary reports of parts of this study have been published previously in abstract form (Granata and Kitai, 1989, 1990).

\section{Materials and Methods}

\section{General procedures}

Male Sprague-Dawley rats (350-380 gm) were anesthetized with a mixture of urethane $(1.8 \mathrm{gm} / \mathrm{kg})$ plus kctaminc $(30 \mathrm{mg} / \mathrm{kg})$. Both agents were intraperitoneally injected. Supplemental doses of ketamine $(30 \mathrm{mg} / \mathrm{kg})$ were administered approximately every $90 \mathrm{~min}$ or when it was necessary in order to maintain the level of anesthesia, which was checked by the suppression of the corneal reflexes, the retraction of the paw, and changes in heart rate in response to pinching of the hind paw. The animals were tracheotomized, and, following paralysis with tubocurarine chloride (initial dose, $0.12 \mathrm{mg} / \mathrm{kg}$, i.m., supplemented with similar dose intravenously every $90 \mathrm{~min}$ ), they were artificially respired with $100 \%$ oxygen with a volume of $250-280 \mathrm{ml} / \mathrm{min}$.

The left femoral artery and femoral vein were cannulated for measurement of arterial pressure and injection of drugs (or lactated Ringer's solution), respectively. Body temperature was maintained at $37^{\circ} \mathrm{C}$ with a thermostatically regulated heating pad connected to a rectal thermistor probc. The rat was placcd in a stereotaxic apparatus (David Kopf Instr.) modified for spinal cord investigation with the bite bar $3.5 \mathrm{~mm}$ below the interaural line and suspended by clamps on the spinal processes of $\mathrm{T}_{6}$ and on the proximal section of the tail to obtain stable recording conditions, which were also improved by a bilateral pneumothorax. An occipital craniotomy and a laminectomy at $T_{1}-T_{3}$ level were performed; then a bipolar stainless steel electrode fabricated from 00 insect pins, insulated with Epoxylite to within $0.2-0.3 \mathrm{~mm}$ from the tip and separated by a distance of $0.3 \mathrm{~mm}$, was stereotaxically placed in the IML region at the $T_{2}-T_{3}$ level. The exposed spinal cord was covered with warm mineral oil. Baroreceptors were constantly activated or inactivated by bolus intravenous injections ( $25 \mu \mathrm{l}$ volume) of phenylephrine $(100 \mu \mathrm{g} / \mathrm{ml}$ dissolved in saline) or sodium nitroprusside $(400 \mu \mathrm{g} / \mathrm{ml}$ in saline), which produced a rise or reduction in arterial pressure, respectively.

In some experiments, in which unparalyzed rats were allowed to ventilate spontaneously, the tracheal pressure was monitored by means of a strain gauge pressure transducer. This signal was used to trigger the averages of the membrane potential of neurons with rhythmic activity 
apparently related to the respiratory cycle. These rhythmically active neurons were also observed under paralysis and artificial ventilation. However, after switching the respirator off for a few seconds, the rhythmic activity was still present, ruling out the possibility that mechanical artifacts from respiratory movements originated the rhythmic activity on these neurons.

\section{Recording and stimulation}

Intracellular recordings were performed with microelectrodes made from borosilicate $(2.0 \mathrm{~mm}$ o.d.) filament glass capillary tubing, filled with a solution of $4-6 \%$ biocytin (Sigma) in $0.05 \mathrm{M}$ Tris buffer, $0.5 \mathrm{M} \mathrm{KCl}$, or $\mathrm{K}$-acetate, $\mathrm{pH} 7.0-7.6$. The resistances of the electrodes measured in saline solution varied from 85 to $160 \mathrm{M} \Omega$. Intracellular potentials were recorded in a conventional manner; they were amplified using a lownoise-high-input resistance bridge amplifier. Intracellular potentials were displayed on a Tektronix ( 8000 series) analog oscilloscope for direct online analysis, simultaneously digitized at rates of $2-50 \mu \mathrm{sec}$ per point ( $16 \mathrm{~K}$ points) at 12 -bit precision by a Nicolet $494 \mathrm{C}$ digital oscilloscope, and stored on floppy disks and on a VCR system for off-line analysis. The amplified DC recordings of neuronal membranc potential, record of current injections, arterial pressure, heart rate, and electrocardiogram (ECG; recorded with subcutaneous chest electrodes) were first digitized with an $\mathrm{A} / \mathrm{D}$ converter $(22 \mathrm{~K}$ samples/sec $)$ and displayed on an electrostatic recorder (Gould 3000 programmable) for on-line analysis. Only neurons with a membrane potential of $-46 \mathrm{mV}$ or more were intracellularly labeled with biocytin by passing 4-6 nA hyperpolarizing rectangular pulses of $150 \mathrm{msec}$ duration at $3.3 \mathrm{~Hz}$ for $3-7 \mathrm{~min}$.

Changes in membrane input resistance occurring during baroreceptor reflex activation were determined by pulses of negative current injected through the recording electrode. The pulses were delivered at constant amplitude and duration. Electrical stimuli (monopolar $0.5 \mathrm{msec}$ cathodal pulses at 50-150 $\mu \mathrm{A}$ ) delivered through bipolar electrodes placed in the IML region at the $T_{1}-T_{3}$ level were used to activate antidromically RVLM neurons with axons that ended in this region or passed near it. The following criteria were used to distinguish antidromic activation of RVLM neurons from orthodromically evoked responses, produced by the stimulation of either spinal afferent fibers or by recurrent collaterals to the RVLM, originating from descending projections terminating or passing through the IML: (1) constant onset latency; (2) at threshold level of stimulation, the responses were all-or-none, and no underlying potentials were observed when the spike failed; (3) high following frequency $(750 \mathrm{~Hz}$ or more); and (4) the collision test (with a spontaneously firing action potential), performed at $2 \times$ threshold intensity of stimulation. A pulse generator (Digitimer) connected to a stimulus isolator was used to deliver the stimulus to the spinal cord. Pulses delivered from spontaneous action potentials were obtained from a window discriminator and used to trigger the Digitimer. As the Digitimer could deliver the stimulus at different selected times after a discriminated spike, the collision test was performed by delaying the pulse applied to the spinal cord with the proper reference to the "critical delay period" (Lipski, 1981).

Data were analyzed with the aid of a digital oscilloscope (Nicolet 4094C) and plotted on an x-y plotter. Signal averages of neuronal membrane potential and pulsatile arterial pressure were constructed off line using the sweep-averaging Nicolet model 4562 . The averages were triggered either by the pulsatile arterial pressure or by the $\mathrm{R}$-wave of the ECG. The data acquisition and analysis program WAVEFORM BASIC (Nicolet Test Instruments Div.) was used to transfer and display data on an IBM AT computer. This program was used to calculate and analyze onset latency of evoked responses, amplitude and duration of action potentials, excitatory postsynaptic potentials (EPSPs), and inhibitory postsynaptic polentials (IPSPs) and to determine the correlation between changes in membrane potential, ECG, and pulsatile arterial pressure. This program was also used to save and recall data on floppy disk.

\section{Histology and immunocytochemistry}

At the end of the experiment, an extra dose of urethane was given (1.8 $\mathrm{gm} / \mathrm{kg}$, i.v.), and the rat was perfused through the heart with heparinized saline solution $(50 \mathrm{ml})$, followed by a mixture of $1 \%$ paraformaldehyde and $2 \%$ glutaraldehyde in $0.1 \mathrm{M}$ phosphate buffer $(\mathrm{pH} 7.4)(500 \mathrm{ml})$. The brain was removed and postfixed overnight in fresh fixative at $4^{\circ} \mathrm{C}$ and then cut into $50 \mu \mathrm{m}$ serial transverse sections through the RVLM by a vibratome and placed in $0.1 \mathrm{M}$ phosphate buffer. After that, sections were processed for histofluorescence of intracellularly labeled neurons according to the method of Horikawa and Armstrong (1988). Briefly, the histological sections were incubated for $4 \mathrm{hr}$ in avidin-Texas red $1: 200$ in phosphate buffer solution plus $0.1 \%$ Triton X-100 and $1 \%$ sodium azide. At the end of the incubation, the tissue was rinsed several times in a fresh solution of phosphate buffer for $1 \mathrm{hr}$ and the sections were mounted in a solution of glycerol in phosphate buffer (1:3) plus $1 \% n$-propyl gallate. Sections were then examined under a Nikon epifluorescence microscope with a rhodamine cube $(\mathrm{G}-2 \mathrm{~A})$.

The histological sections containing intracellularly labeled neurons (fluorescent images) were photographed and then processed for phenylethanolamine- $N$-methyltransferase (PNMT) immunohistochemistry. The polyclonal antiserum was produced in rabbits against PNMT obtained and purified from bovine adrenal medulla and analyzed for immunological specificity according to Joh and Goldstein (1973).

Histological sections were rinsed in phosphate buffer and incubated in 3\% normal goat serum and $0.2 \%$ Triton X-100 in phosphate buffer at room temperature. After that, sections were incubated in a 1:2000 dilution of PNMT antiserum plus $0.1 \%$ Triton $X-100$ at $4^{\circ} \mathrm{C}$ for $18 \mathrm{hr}$ In four experiments, instead of using a PNMT antiserum, an antiserum against tyrosine hydroxylase (TH) was used. Following that, sections were washed several times in phosphate buffer $(45 \mathrm{~min})$ and incubated sequentially with a 1:50 dilution of fluorescein isothiocyanate (FITC)conjugated goat anti-rabbit immunoglobulin ( $\mathrm{IgG}$ ) for $2 \mathrm{hr}$ at room temperature. Incubations were done with continuous agitation. Sections were finally rinsed in phosphate buffer for $45 \mathrm{~min}$ and mounted on gelatin-coated glass microscope slides and coverslipped with glycerol in phosphate buffer (1:3) containing $1 \%(\mathrm{v} / \mathrm{v}) n$-propyl gallate.

Histofluorescent examination for double-labeled neurons. Sections were examined under an epifluorescence microscope for possible double labeling of neuronal cell bodies and neuronal processes. The biocytininjected neurons were examined with a G-2A filter (green) and immunostained neurons with a B-2A filter (blue). The FITC fluorescence is not seen through the G-2A filter; however, neurons well filled and intensely stained with biocytin-avidin-Texas red complex will produce a weak orange-red color through the blue filter. This phenomenon is less apparent in proximal dendrites, which were used to rule out possible false-positive double labeling. However, in some neurons this possibility was further investigated by changing the immunohistofluorescence reaction to routine light microscopy using the peroxidase-antiperoxidase (PAP) method of Sternberger (1979). The histological sections were incubated in rabbit PAP $(1: 80)$ complex in phosphate buffer plus $2 \%$ normal goat serum for $2 \mathrm{hr}$. After that, sections were rinsed in phosphate buffer for $45 \mathrm{~min}$, and the PAP reaction product was shown by incubation with $0.05 \% 3,3^{\prime}$-diaminobenzidine (DAB) and $0.03 \% \mathrm{H}_{2} \mathrm{O}_{2}(\mathrm{pH}$ 7.4). Sections were mounted in buffered glycerol and examined under light microscopy.

Histological analysis of stimulation sites. In some experiments, stimulation sites in the IML in the thoracic spinal cord were determined by marking at the completion of the experiment the electrode tip location. To do that, $1 \mathrm{~mA}$ intensity positive current was passed for $15 \mathrm{sec}$ and $40 \mu \mathrm{m}$ coronal sections of the spinal cord were stained with cresyl violet. Simulation sites were mapped and drawn with a camera lucida, under a light microscope.

Results are expressed as means \pm standard deviation (SD) or standard error (SE) of the mean.

\section{Results}

\section{Identification and characterization of RVLM neurons with baroreceptor-related activity}

The RVLM was systematically investigated for spontaneously active neurons with action potentials occurring at about every cardiac cycle. This first approach was done at an extracellular level. Figure $1 A$ shows the extracellular action potentials of a neuron located at $-3.4 \mathrm{~mm}$ from the interaural line and at 200 $\mu \mathrm{m}$ from the ventral surface of the medulla. The visual examination of the neuronal ongoing activity demonstrated that this RVLM neuron discharged action potentials about every cardiac cycle, as depicted in a typical simultaneous recording of the pulsatile arterial pressure and the extracellular recording (Fig. $1 A$ ). This examination was carried on for at least 20 sweeps, with the aid of a storage oscilloscope triggered by the pulsatile 

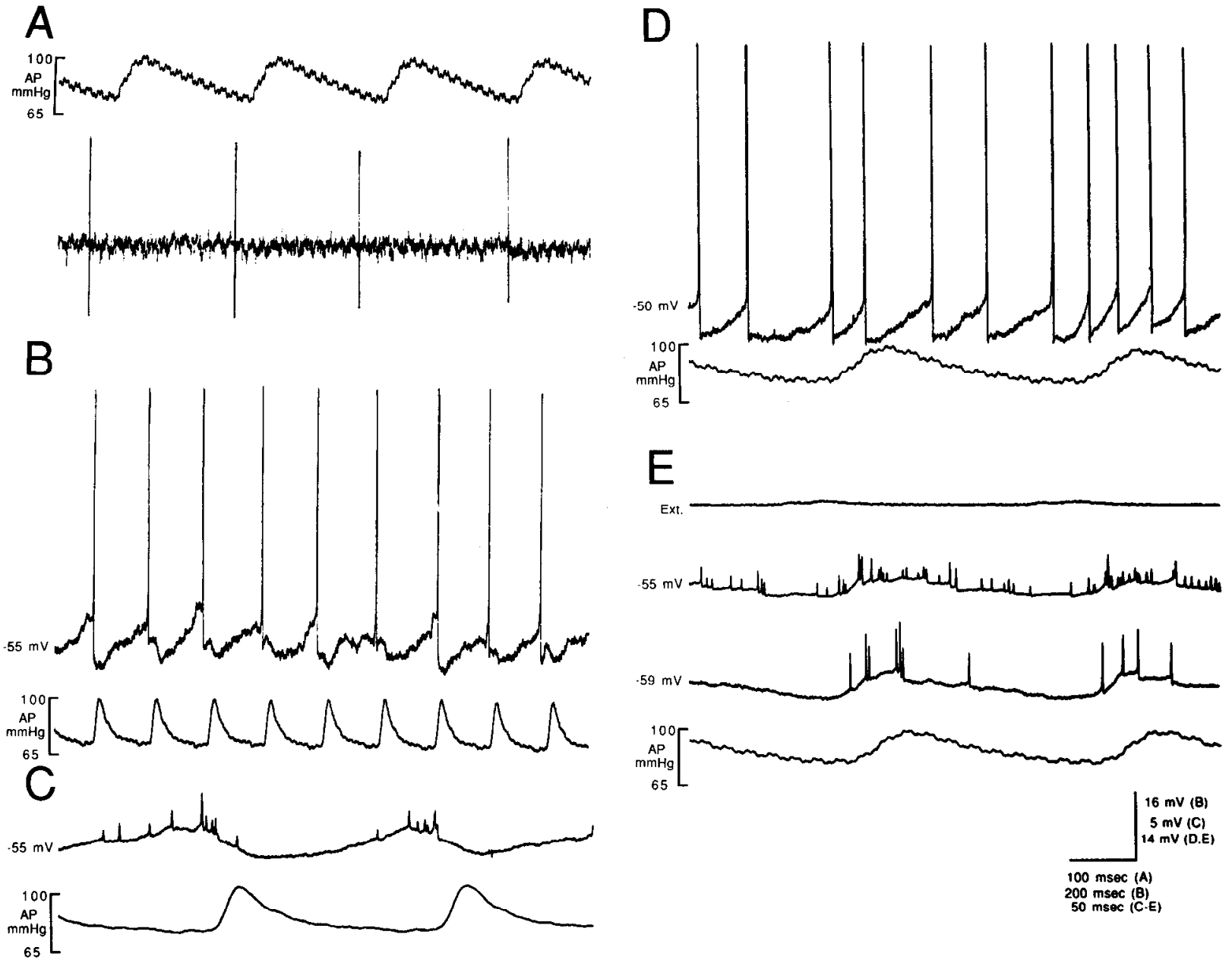

Figure 1. Extracellular and intracellular recordings from different bulbospinal RVLM neurons with cardiac-related activity. $A$, Digitized record of extracellular recording of a spontaneously active RVLM neuron and arterial pressure $(A P)$ in $\mathrm{mm} \mathrm{Hg}$. $B$, Intracellular recording of the same neuron as in $A$ at stable resting membrane potential $(-55 \mathrm{mV})$. The neuron has recovered its pulse-related firing pattern observed in $A$. The digitized records of arterial pressure and membrane potential were simultaneously triggered by the pulsatile arterial pressure. $C$, Simultaneous average of the neuronal membrane potential and pulsatile arterial pressure at resting values. The averages were triggered by the arterial pulse wave. Number of sweeps was 450 . Sample period, $50 \mu \mathrm{sec}$. $D$. Simultaneous digitized records of arterial pressure $(\mathrm{mm} \mathrm{Hg})$ and intracellular recording of a different neuron at stable membrane potential $(-50 \mathrm{mV})$ triggered by the arterial pulse wave. Note the increased frequency of discharge at the end of diastolic periods. $E$, Simultaneous average of the arterial pressure in $\mathrm{mm} \mathrm{Hg}$ at resting values and the spontaneous neuronal membrane potential (upper middle trace) and the neuronal membrane potential held at $-59 \mathrm{mV}$ (lower middle trace). The average of the extracellular (Ext.) recording is illustrated in the uppermost trace. The averages were triggered by the arterial pulse wave. Same neuron as in $D$. Number of sweeps was 550 in all cases. Sample period, $50 \mu \mathrm{sec}$.

arterial pressure. This procedure was conducted in order to test the consistency of the cardiac-related activity. Neurons like this, as well as others with a similar pattern of discharge, were selected for intracellular analysis. After penetration, when the recording conditions are improved and the membrane potential reached stable values $(-55 \mathrm{mV})$, the neuron spontaneously depolarized and discharged action potentials at practically every cardiac cycle (Fig. $1 B$ ). In order to investigate further the cardiac-related rhythmic activity of this RVLM neuron, we analyzed the relationship between the neuronal membrane potential and the pulsatile arterial pressure. Figure $1 C$ illustrates the simultaneous average of the neuronal membrane potential and pulsatile arterial pressure triggered by the arterial pulse wave ( 550 sweeps). The average fluctuations of membrane potential correlated with the period of the cardiac cycle. Slow depolarization increased gradually during the middiastolic phase and reached maximal values at the beginning of the systolic period. The depolarization was followed by hyperpolarization. The cardiac-related rhythmic activity observed in this type of neuron is shown in another case in Figure 1, $D$ and $E$. At resting membrane potential, the spontaneously occurring intracellular action potentials were somewhat irrcgular; however, it is possible to observe a faster firing rate at the end of the diastolic/beginning of the systolic period (Fig. 1D). The average of the neuronal membrane potentials triggered by the arterial pressure pulse demonstrated depolarization potentials occurring at similar times in the cardiac cycle (Fig. $1 E$ ). This depolarizing potential increased in magnitude when the neuron was hyperpolarized by passing negative current through the recording electrode; the membrane potentials determined at similar times of the cardiac cycle were $-55 \mathrm{mV}$ before and $-59 \mathrm{mV}$ after hyperpolarization (Fig. $1 E$, lower trace). In the upper trace of this figure is illustrated the average of the extracellular recordings obtained immediately after the electrode was withdrawn from inside the neuron but 


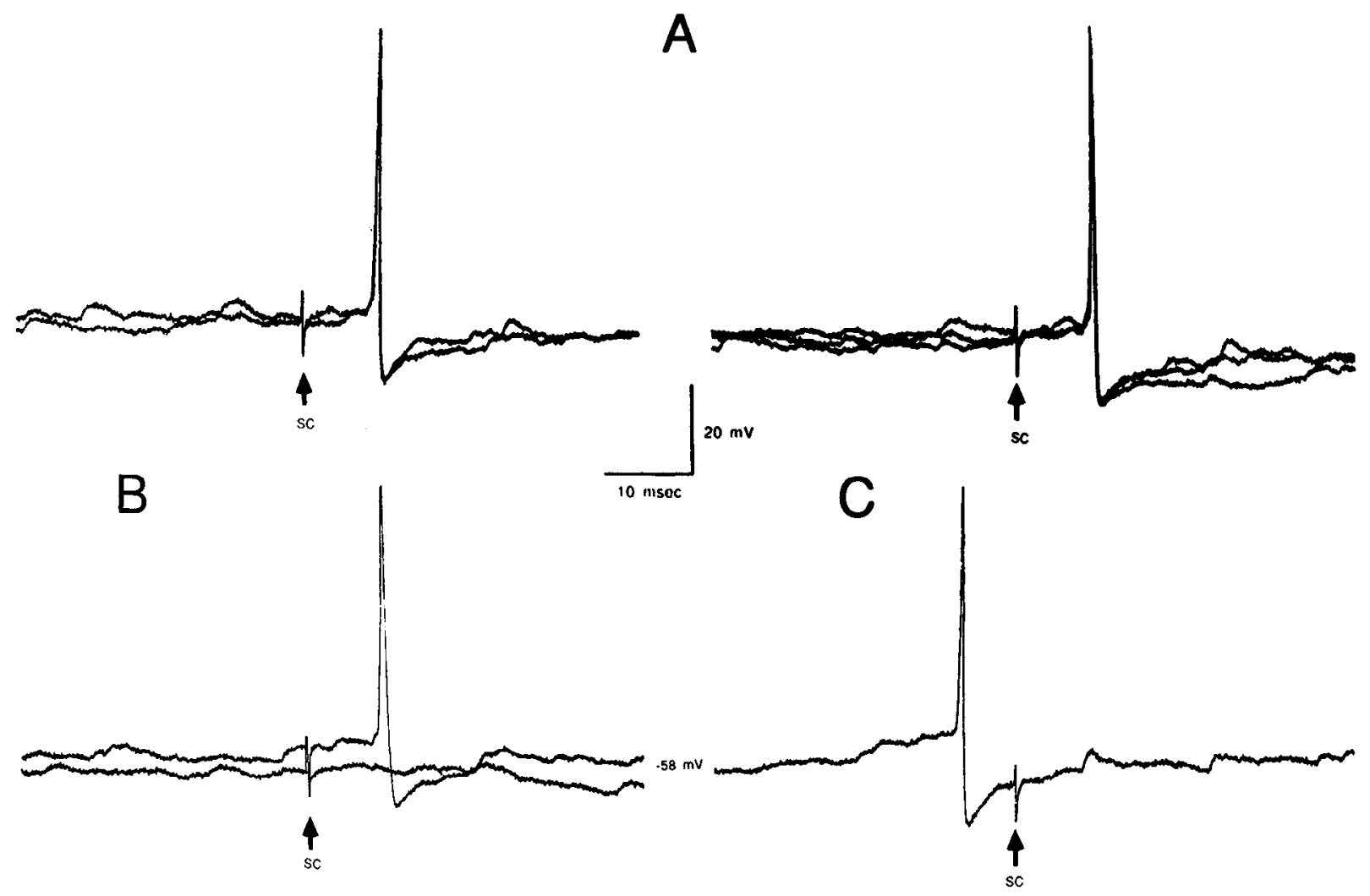

Figure 2. Antidromic activation of a RVLM neuron with pulse-modulated activity following spinal cord stimulation. In $A$, electrical stimulation of the IML in the ipsilateral spinal cord $(s c$, arrow) during intracellular recording produced action potentials, which demonstrated constant latency. In $B$, at stimulation threshold $(75 \mu \mathrm{A})($ arrow), spikes were all-or-none, and no underlying EPSPs were observed when the spike failed (the DC trace is shifted to improve visualization). In $C$, a spontaneous spike collided with the antidromically elicited action potential at $2 \times$ threshold of stimulation (arrow).

was still very close to it. Unlike the intracellular average, the resulting extracellular average was flat.

The absence of fluctuations in the extracellular average would indicate that mechanical artifacts (sometimes very conspicuous in this part of the brain) were insufficient to interfere with the intracellular potentials.

\section{Antidromic activation of RVLM-spinal neurons}

To investigate whether neurons located in the RVLM with modulatory inputs from baroreceptors send descending projections to the spinal cord, the recorded neurons were systematically tested for antidromic activation by electrical stimulation of the IML of the ipsilateral thoracic spinal cord $\left(\mathrm{T}_{2}-\mathrm{T}_{3}\right.$ level). Wellestablished criteria, already explained in Materials and Methods, were used for the evaluation of antidromically evoked responses. The results obtained in a typical barosensitive neuron that was activated at a constant latency by IML stimulation at $1.5 \times$ threshold are shown in Figure $2 \mathrm{~A}$. When this neuron was stimulated at the threshold level, the evoked responses were allor-none, and no underlying potentials were observed when the spike failed (Fig. $2 B$; here the DC is shifted to improve visualization). The collision test for antidromic activation is shown in Figure $2 C$; a spontaneous spike occurring during the critical delay period collided with the antidromic evoked action potential. The IML was stimulated at $2 \times$ threshold.

Twenty-five barosensitive neurons were activated antidromically. The calculated conduction velocities of the RVLM de- scending axons varied from $1.5 \mathrm{~m} / \mathrm{sec}$ to $11.0 \mathrm{~m} / \mathrm{sec}$. The mean value was $5.5 \pm 2.6 \mathrm{~m} / \mathrm{sec}(\mathrm{SD})(n=25)$.

\section{Baroreceptor reflex responses of RVLM bulbospinal neurons}

The synchronization to the cardiac cycle of the excitatory potentials observed in this type of RVLM bulbospinal neuron indicates that inputs originating in the baroreceptors affected the activity of these neurons. This phenomenon was observed at values of resting mean arterial pressure below $95-100 \mathrm{~mm}$ $\mathrm{Hg}$. From these results, however, the importance of the influence of the baroreflex inputs in governing the total activity of this type of neuron is still unclear. We therefore decided to examine the influence of baroreceptor reflex activation or inactivation on the firing frequency, on the membrane potential, and, when possible, on the membrane input resistance of RVLM bulbospinal neurons. To assess the influence of baroreceptor reflex activation on these neurons, the systemic arterial pressure was transiently increased by bolus intravenous injections of phenylephrine. Despite the high incidence of neuronal damage produced by the movements associated with the abrupt changes in arterial pressure, we nevertheless succeeded in testing the effect of manipulating baroreceptors on three medullospinal neurons with rhythmic fluctuations entrained to the cardiac cycle. The increases in mean arterial pressure ranged between 18 and 25 $\mathrm{mm} \mathrm{Hg}(n=3)$, while the hyperpolarization produced by these changes in arterial pressure varied from -2.6 to $-7.0 \mathrm{mV}(n$ $=3$ ). The increases in input resistance ranged between $13 \%$ and 

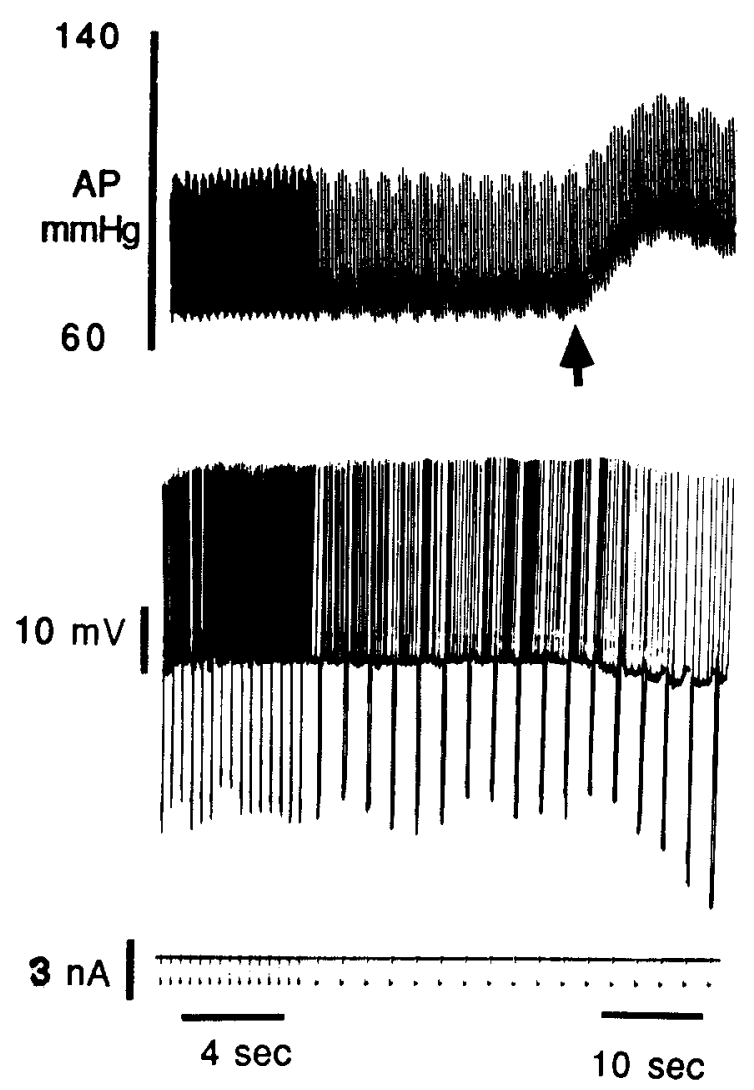

Figure 3. Inhibition of a bulbospinal RVLM neuron by phenylephrineproduced increases in systemic arterial pressure $(A P)$. Intravenous administration of a bolus injection of phenylephrine (arrow) during intracellular recording from a spinal projection neuron in the RVLM elicited (1) hyperpolarization of the neuronal membrane with a decrease in the firing frequency, and (2) an increase in the input resistance, as demonstrated by the increased amplitude of hyperpolarizing voltage deflections in response to constant-amplitude inward current pulses. From top to bottom, systemic arterial pressure $(\mathrm{mm} \mathrm{Hg})$, intracellular recording (membrane potential, $-50 \mathrm{mV}$ ), and constant current injections. Note the different horizontal calibration at the beginning of the recording. All spikes are attenuated in size by the pen recorder.
$38 \%(n=3)$. In one neuron, an increase in arterial pressure of $55 \mathrm{~mm} \mathrm{Hg}$ was accompanied by an increase in the input resistance of $50 \%$; however, the maneuver produced an irreversible damage in the neuron. Figure 3 shows the pressor response elicited by a bolus injection of phenylephrine accompanied by hyperpolarization of the neuronal membrane potential and a decrease in the firing rate. The input impedance, as determined by deflections in the membrane potential by constant pulses of negative current, was increased during the hyperpolarization. Driving the membrane potential up to similar levels observed during the increases in arterial pressure did not produce changes in input impedance (not shown). The neuronal input resistance was calculated in five neurons as the slope of the regression line derived from the plot of the current injections versus the deflections in membrane potential; these values ranged between 10.6 and $14.5 \mathrm{M} \Omega$.

The influence of baroreceptor inactivation on these RVLM neurons was tested by reducing systemic arterial pressure by bolus intravenous injections of the vasodilator sodium nitroprusside. The drop in arterial pressure produced by this drug (Fig. 4) elicited a depolarizing response with an increase in the firing frequency. To rule out the possibility of neuronal damage due to the movement produced by this experimental procedure, a constant current $(-0.2 \mathrm{nA})$ was intracellularly injected (Fig. 4). During this period, the neuronal membrane potential and the frequency of discharge were both reduced. However, when the passage of current was interrupted, the membrane potential resumed a prepassage of current relationship to the arterial pressure. In those neurons damaged by this procedure, an irreversible depolarization was observed.

\section{Recording from noncardiovascular neurons}

Intracellular potentials were obtained from a different type of neuron commingling with the previously described group in the RVLM $(n=12)$. These neurons were also antidromically activated from the spinal cord with axonal conduction velocities slower than $12 \mathrm{~m} / \mathrm{sec}$. They displayed a regular pattern of discharge (Fig. 5A). Brief hyperpolarization of the neuron, up to below spike threshold level, did not modify the subsequent firing
Figure 4. Influence of sodium nitroprusside-induced hypotension on a bulbospinal RVLM neuron with pulsemodulated activity. Intravenous administration of a bolus injection of sodium nitroprusside (arrow) during intracellular recording from a bulbospinal RVLM neuron reduced systemic arterial pressure $(A P)$, causing neuronal depolarization and an increase in the firing rate. To check that the neuron was not damaged by this procedure, the membrane potential was hyperpolarized by constant negative current injection of $-0.2 \mathrm{nA}$ for a period illustrated in the figure. When the current injection was interrupted, the membrane potential recovered a proportional value according to the arterial pressure at that moment. The spikes are attenuated in size by the pen recorder.

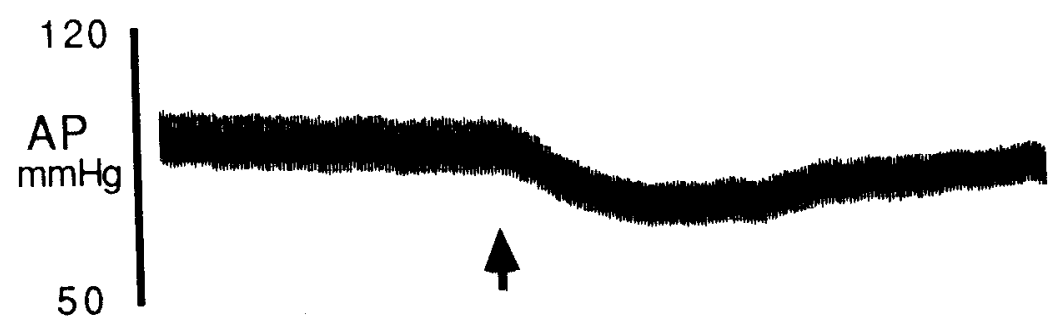

$-58$

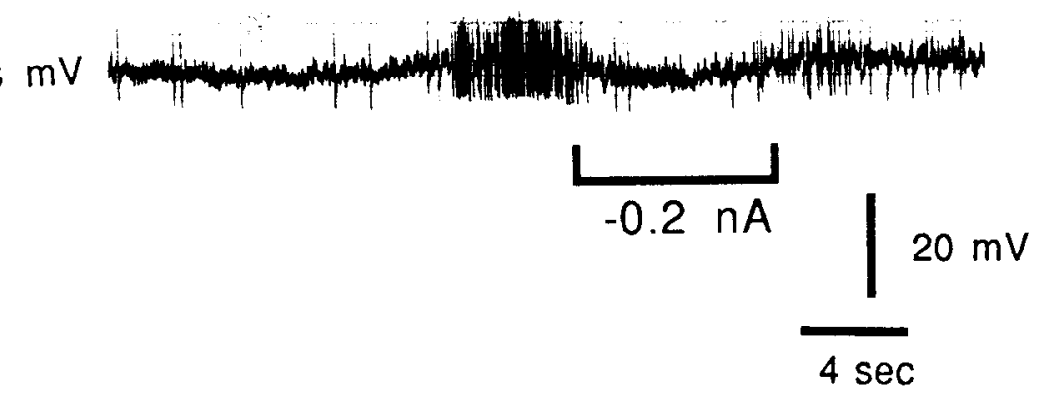



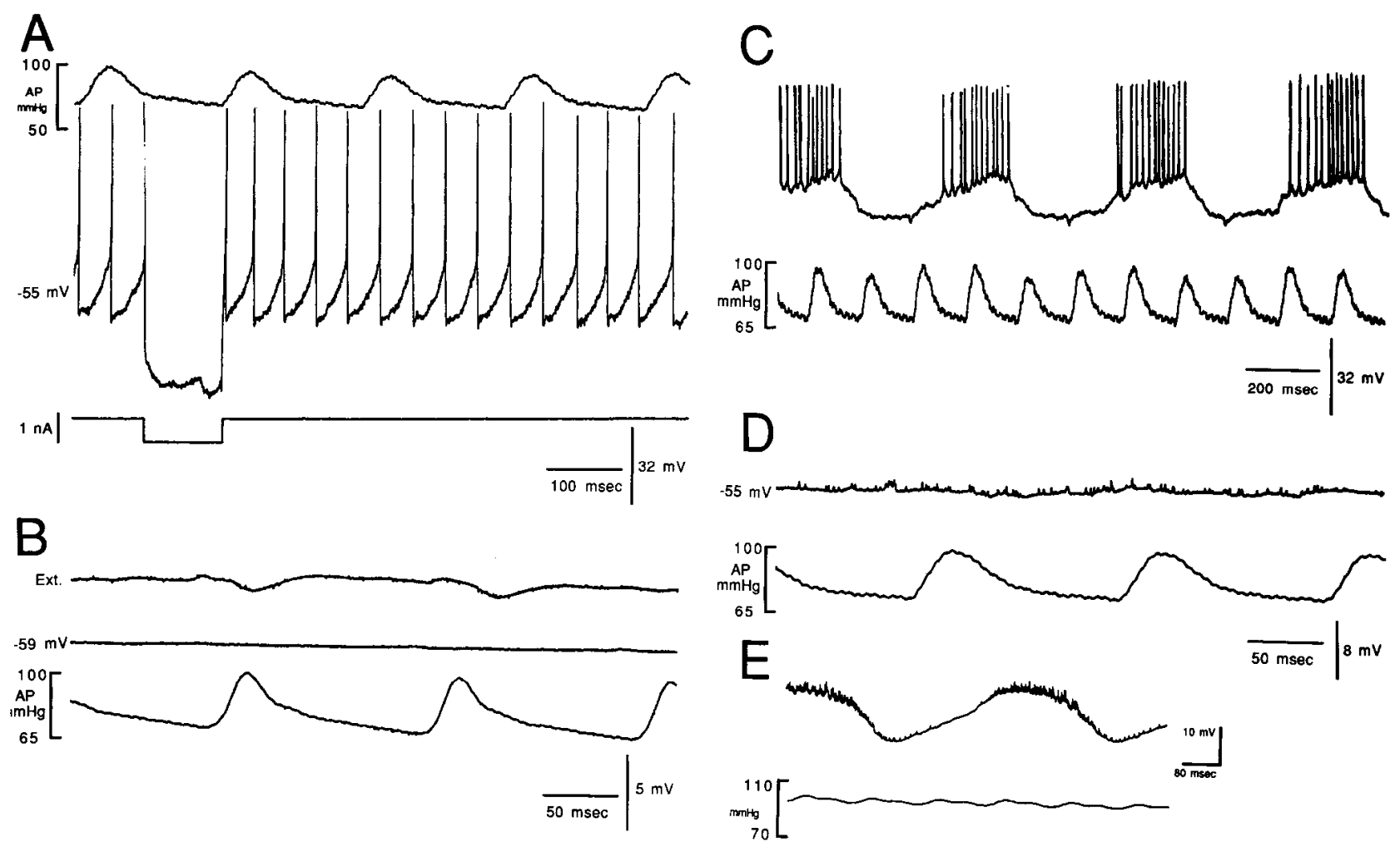

Figure 5. Intracellular recording from different RVLM neurons with very regular or rhythmic fluctuation activity, but not entrained to the arterial pulse. $A$, Simultaneous digitized records of pulsatile arterial pressure $(A P)$ in $\mathrm{mm} \mathrm{Hg}$ and intracellular recording from a $\mathrm{RVLM}$ bulbospinal neuron at stable membrane potential and normal values of arterial pressure. The records were triggered simultaneously by the arterial pulse wave. A 100 msec duration pulse of negative current was intracellularly applied. The intensity of the current injected is indicated at the bottom of the trace. $B$, Simultaneous average of the pulsatile resting arterial pressure and the neuronal membrane potential (middle trace). The average of the extracellular recording $(E x t$.) is illustrated in the upper trace. All three averages were triggered by the arterial pulse wave. Number of sweeps was 620 . Sample period, $50 \mu \mathrm{sec}$. $C$, Intracellular recording from a different RVLM neuron with phasic depolarization and pulsatile arterial pressure. $D$, Simultaneous averages of the pulsatile arterial pressure and the neuronal membrane potential $(-55 \mathrm{mV})$. The averages were triggered by the arterial pulse wave. Same neuron as in $C$. Number of sweeps was 720 . Sample period, $50 \mu \mathrm{sec}$. $E$, Simultaneous averages of the pulsatile arterial pressure and the membrane potential of a neuron with similar pattern of discharge to thosc described in $C$ and $D$. The averages were triggered by the tracheal pressure in an unparalyzed rat breathing spontaneously. Number of sweeps was 360 . Sample period, $50 \mu \mathrm{sec}$.

rate (Fig. 5A). Moreover, close examination demonstrated a delay in the occurrence of the first spike following the current injection in relation to the spike that could have occurred in the absence of that injection. In addition, a flat average of the neuronal membrane potential triggered by the arterial pressure pulse was observed (Fig. $5 B$, middle trace). The average of the extracellular recordings in this neuron demonstrated some movement artifacts, which were again unable to affect the intracellular recording. Therefore, unlike that of the neuronal type described in Figures 1-4, the activity of these RVLM bulbospinal neurons demonstrated a very regular pattern of discharge, and the membrane potential of these neurons did not demonstrate rhythmic fluctuations synchronized with the arterial pulse.

In this study, we recorded the neuronal activity from a group of neurons located in the more dorsal and caudal aspect of the RVLM. These neurons were antidromically activated from the thoracic spinal cord with intensities of stimulation above 200 $\mu \mathrm{A}$, and the axonal conduction velocities varied between 16.6 and $33.0 \mathrm{~m} / \mathrm{sec}$. At resting conditions, the membrane potential of these neurons demonstrated a rhythmic discharge pattern; however, the phasic character of their depolarization and discharge was not related to the cardiac cycle (Fig. $5 \mathrm{C}$ ). On the other hand, a correlation of the membrane potential rhythm with the respirator cycle was obscrved. To rulc out the possibility that mechanical artifacts due to the ventilator could have produced the rhythmic activity on these neurons, the ventilator was shut off for a few seconds; under these conditions the rhythmic neuronal activity was still present. These rhythmically active neurons not related to cardiac cycle were also found in unparalyzed, spontaneously breathing rats. The average of the neuronal membrane potential triggered by the tracheal pressure showed that the rhythmic activity of these neurons was synchronized with the respiratory cycle (Fig. $5 E$ ). Since the phrenic nerve activity was not recorded in these experiments, we are not in a position to relate these neurons clearly to the generation or transmission of central respiratory activity. However, the present results are consistent with this idea, as demonstrated by others using extracellular recording techniques (Howard and Tabatabai, 1975). The average of the neuronal membrane potentials triggered by arterial pressurc pulse was flat in these RVLM neurons (Fig. $5 D$ ). These results, first, demonstrated the absence of baroreceptor modulation in these neurons that may be involved in respiratory function and, second, proved again in a different type of RVLM neuron the lack of mechanical artifacts related to arterial pulsation in the present experimental conditions. 

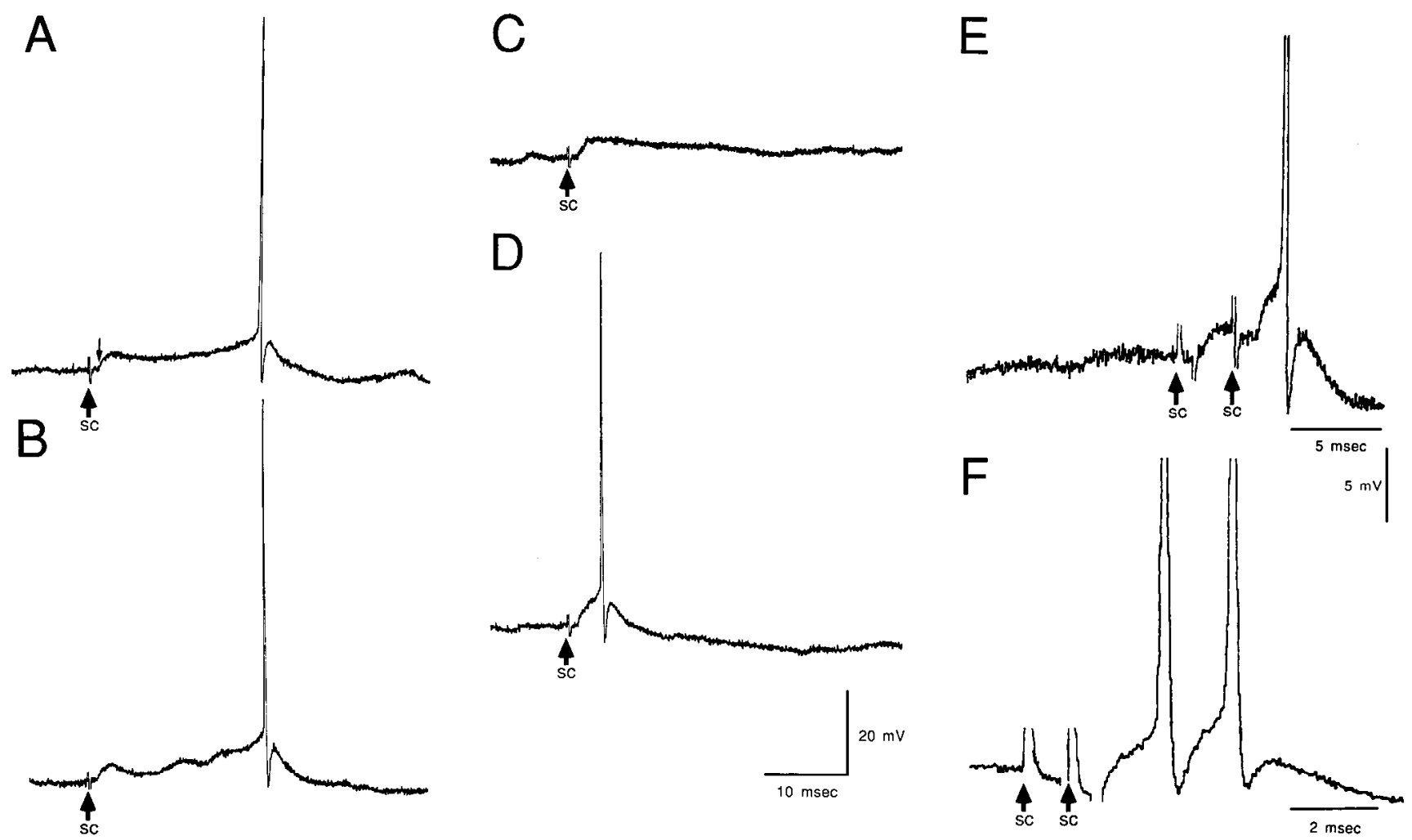

Figure 6. Orthodromic and antidromic responses of a RVLM neuron following spinal cord stimulation. In $A$ and $B$ are shown two consecutive antidromic spike potentials evoked by electrical stimulation of the ipsilateral IML in the spinal cord (sc, arrows). The constant onset latency antidromic spikes were preceded by a very short onset latency EPSPs (downward small arrow in $A$ ). In $C$, the antidromic action potential was allor-none, and no underlying potentials were observed when the action potential failed at threshold level of stimulation. In $D$, when the intensity of spinal cord stimulation (arrow) was increased, the EPSP generated an action potential that collided with the antidromically elicited action potential. Note the absence of underlying potentials when the antidromic spike failed. Calibration is shown for $A-D$. In $E$, repetitive stimulation with two consecutive pulses at $333 \mathrm{~Hz}$ and with the same intensity (arrows) produced summation of the evoked monosynaptic EPSPs without variations in the onset latency in the same neuron. Note the second evoked EPSP reached threshold for spike generation. In $F$, two consecutive pulses at 1000 $\mathrm{Hz}$ and again with the same intensity (arrows) evoked two EPSPs that generated two action potentials.

\section{Inhibitory baroreceptor inputs on bulbospinal RVLM neurons}

A different type of neuron located in the RVLM was identified in this study on the basis of the pattern of discharge and responses to IML stimulation. Figure $6, A$ and $B$, illustrates the response to spinal cord stimulation in a typical example of a neuron in which an antidromic action potential with constant onset latency is evoked at $1.5 \times$ the threshold level of stimulation. The axonal conduction velocities of this type of neuron varied between 1.5 and $3.1 \mathrm{~m} / \mathrm{sec}(n=8)$. At the threshold level of stimulation for the antidromic spike, this was all-or-none; no underlying potentials were observed when the action potential failed, as shown in Figure $6 \mathrm{C}$. In addition, in six of eight neurons, an EPSP with a very short onset latency preceded the antidromic response. In Figure 6, $A-C$, the preceding EPSP was evoked with a latency of $1.5 \mathrm{msec}$, and the amplitude was $3.1 \mathrm{mV}$. The intensity of current shown in Figure $6, A-C$, was below that needed to evoke an EPSP large enough to produce an action potential. However, when the intensity of spinal cord stimulation was increased and the EPSP produced an action potential, this collided with the antidromic spike (Fig. $6 D$ ). Note that no underlying potentials were present when the antidromic potential collided. Figure $6 E$ shows the same EPSP producing summation without variations in the onset latency when the spinal cord was stimulated with a frequency of $333 \mathrm{~Hz}$. Moreover,
Figure $6 F$ shows these EPSPs can follow very high frequency; in this case, the spinal cord was stimulated with two pulses at $1000 \mathrm{~Hz}$, and both EPSPs produced action potentials. These results demonstrate that these EPSPs were monosynaptically evoked and synaptic transmission is very secure.

Thesc ncurons werc spontancously active, and the action potentials were triggered by slow depolarizing potentials. Therefore, it was very difficult to determine the level of the resting membrane potential. We considered that this value should be close to the potential reached at the beginning of the slow depolarizing potential, when the conductance that mediates this potential is still weak. At membrane potentials of -49 to -53 $\mathrm{mV}$ (calculated as mentioned) and resting values of arterial pressure and heart rate, this type of neuron showed a spontaneous firing pattern consisting of a slower single spiking at the end of the diastolic/beginning of the systolic period, followed by a faster single spiking during the rest of the cardiac period (Fig. 7A). The average of the neuronal membrane potentials triggered by spontaneous spikes showed more clearly this phasing pattern of firing (Fig. $7 B$ ), as well as a shorter period for diastolic spikes and a longer period for systolic spikes. Note the larger number of diastolic spikes during an equal period of time, due principally to the higher frequency of discharge during the diastolic period. The average of the neuronal membrane potentials triggered by the pulsatile arterial pressure on the same neuron demonstrated 

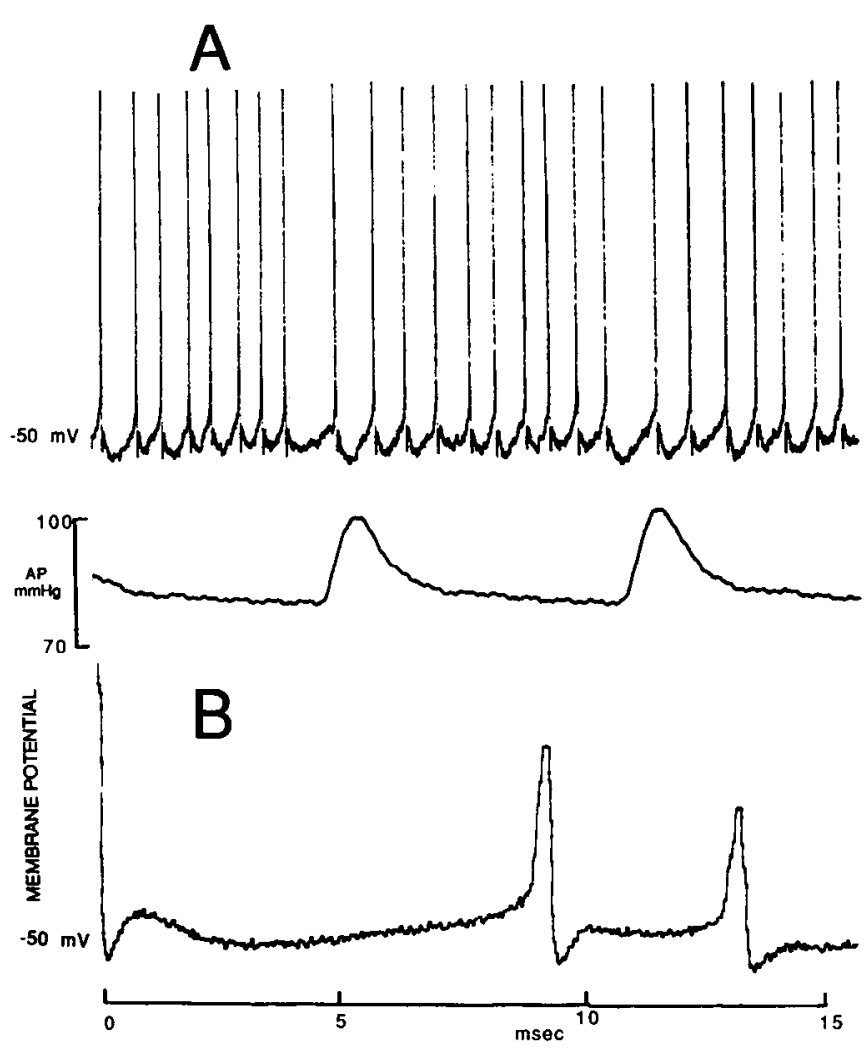
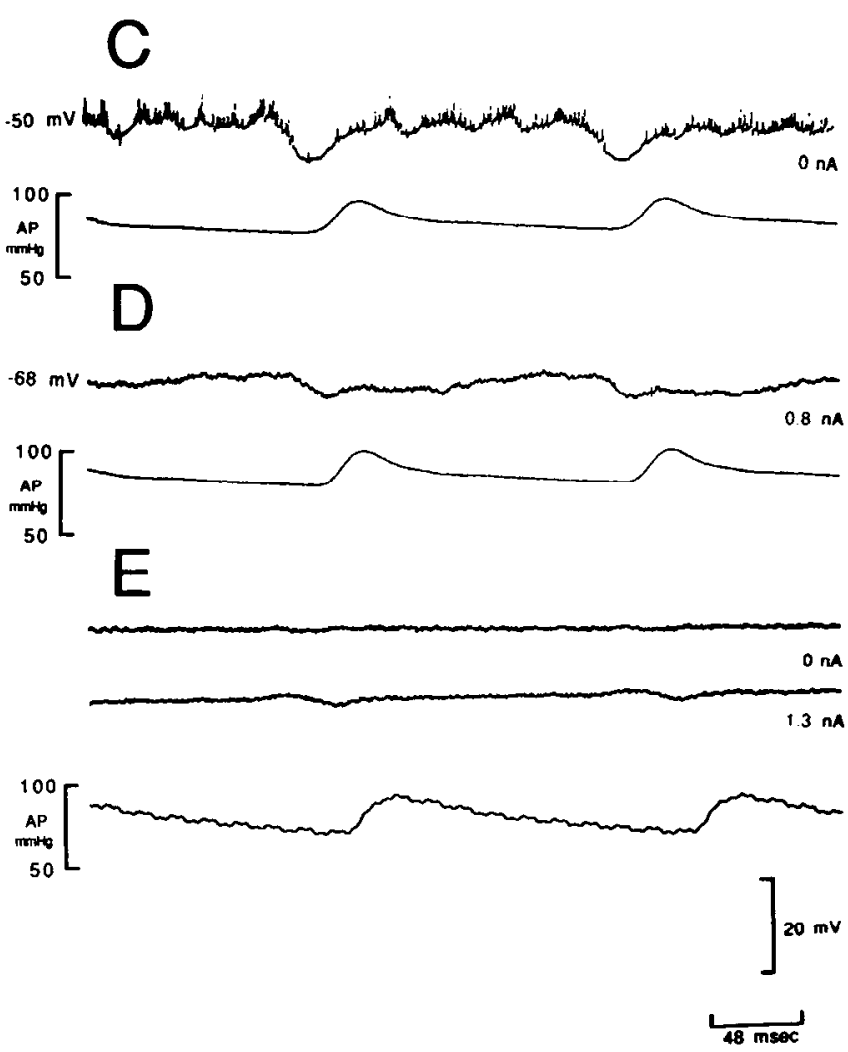

Figure 7. Intracellular recording of a RVLM bulbospinal neuron with inhibitory baroreceptor inputs. $A$, Simultaneous digitized records of arterial pressure $(A P)$ in $\mathrm{mm} \mathrm{Hg}$ and intracellular recording at stable membrane potential $(-50 \mathrm{mV})$. The records were triggered by the arterial pulse wave. Note the decrease in the firing frequency at the end of the diastolic period. $B$, Average of the resting neuronal membrane potentials triggered by spontaneous spikes. Sweeps, 96; sample period, $5 \mu \mathrm{sec}$. $C$, Simultaneous averages of the neuronal membrane potential (-50 mV) and pulsatile arterial pressure in $\mathrm{mm} \mathrm{Hg}$, triggered by the arterial pulse wave. Sweeps, 674; sample period, $50 \mu$ sec. $D$, Averages similar to those illustrated in $C$, but now at a holding membrane potential of $-68 \mathrm{mV}$. $E$. The simultaneous averages of arterial pressure and extracellular recordings at zero or $-1.3 \mathrm{nA}$ constant current injection (upper and middle traces, respectively). The averages were triggered by the arterial pulse wave. Sweeps, 700 ; sample period, $50 \mu \mathrm{sec}$. Same neuron as in $A-D$.

at normal values of arterial pressure and heart rate a rather fast hyperpolarizing potential occurring at the end of the diastolic/ beginning of the systolic period (Fig. 7C). When the neuron was hyperpolarized by intracellular injection of hyperpolarizing current $(-0.8 \mathrm{nA})$, the magnitude of these inhibitory potentials decreased (Fig. $7 D$ ). An equal number of sweeps of extracellular recording, with the tip of the electrode still close to the neuron, resulted in a flat average (Fig. $7 E$, upper tracc), and only minor mechanical distortions were observed when averaging of the extracellular recordings was performed under constant injection of negative current through the recording electrode with a magnitude of $-1.3 \mathrm{nA}$ (Fig. $7 E$, middle trace). This intensity of current was very much higher than the maximum injected during intracellular recording. Figure $8, A$ and $B$, shows, in a neuron similar to the one described in Figures 6 and 7, that shorlly after hyperpolarizing the neuron by passing constant negative current through the recording electrode filled with $\mathrm{KCl}$, the frequency of discharge decreased, but the differences in frequency of discharge between the end of diastolic/early systolic period and the middle of the diastolic period were still present (Fig. 8A). However, it was observed that after injecting pulses of negative current $(-2 \mathrm{nA}, 200 \mathrm{msec})$ for several minutes and holding the membrane potential at more negative values, the inhibitory potentials locked to the cardiac cycle were reversed in polarity (Fig. $8 B$ ). In addition, depending on the holding membrane potentials, these reversed potentials occurring at every cardiac cycle produced action potentials (Fig. $8 B$, upper trace). These results then indicate that these inhibitory potentials synchronized to the cardiac cycle are chloride-dependent IPSPs. Figure $8 C$ illustrates the reversed IPSPs occurring at the end of every diastolic/beginning of systolic period, and, in Figure $8 D$, at a more depolarized neuronal membrane potential level, the reversed IPSPs reached the threshold for spike generation.

\section{Neuronal characterization during hypotension}

The firing pattern described in previous paragraphs and Figures 7 and 8 was identified under normal values of arterial pressure and heart rate. However, these neurons completely changed their firing pattern when systemic arterial pressure was constantly reduced by intravenous infusions of sodium nitroprusside. The pattern of discharge during hypotension consisted of a very regular pacemaker-like activity (Fig. 9A). The spontaneous action potentials were preceded by slow depolarization and were followed by an afterhyperpolarization (Fig. 9B). The afterhyperpolarization that followed each action potential was on some occasions difficult to put in evidence because of the slow depolarization that normally controls the interspike neuronal activity. It is known that in other neurons, the afterhyperpolarization follows single or multiple spikes. Moreover, depolarizing current pulses injected in dopaminergic neurons in 

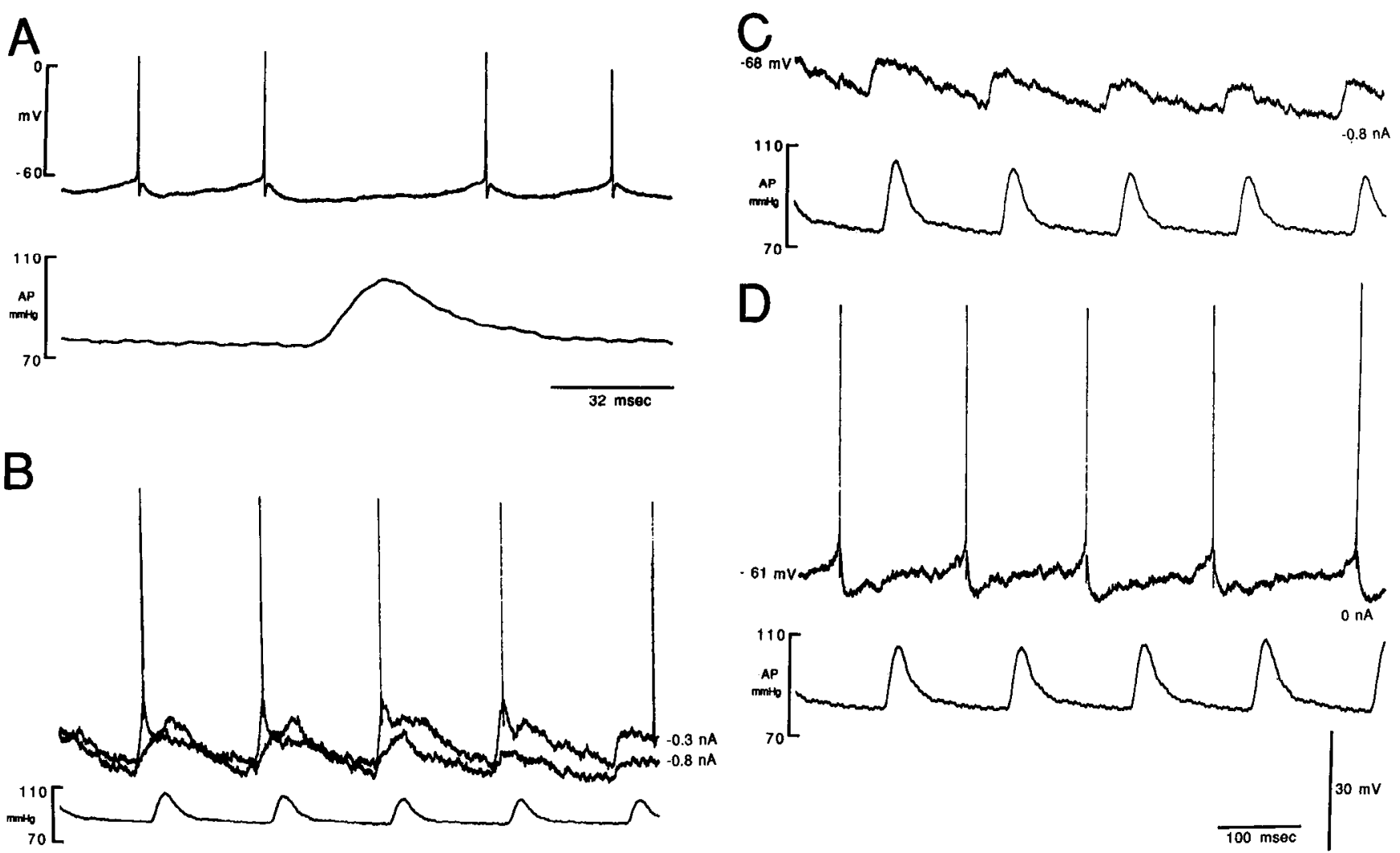

Figure 8. Intracellular rccordings of a RVLM bulbospinal ncuron with inhibitory barorcccptor inputs during intracellular injection of negative current. $A$, Simultaneous records of arterial pressure $(A P)$ and intracellular recording, immediately after holding the membrane potential at more negative values. The records were triggered by the arterial pulse wave. $B$, Similar records as in $A$ (note different time base) after intracellular injections of negative current for several minutes and holding the membrane potential with $-0.3 \mathrm{nA}$ or $-0.8 \mathrm{nA}$ negative current (microelectrode filled with $\mathrm{KCl}$ ). $C$, Simultaneous records of arterial pressure and neuronal membrane potential under similar experimental conditions as in $B$, at a holding membrane potential of $-68 \mathrm{mV}$. $D$, Similar record as in $C$, but at a membrane potential of $-61 \mathrm{mV}$. Same neuron as in $A-C$.

the rat substantia nigra, large enough to trigger action potentials, are followed by an afterhyperpolarizing potential, which is proportional in amplitude to the number of spikes evoked by such depolarizing pulses (Grace, 1987). In RVLM neurons, it was found that the amplitude of the afterhyperpolarization potential that followed depolarizing current pulses was proportional to the number of spikes evoked during the current pulses (Fig. $10 \mathrm{~A}$ ). We did not investigate maximal values attained by these afterhyperpolarizing potentials. However, these results demonstrate the presence of afterhyperpolarization in these RVLM neurons. The slow depolarization was demonstrated to be voltage dependent, as its slope increased with depolarization of membrane potential and decreased during hyperpolarization. Figure $10 D$ illustrates the increments in the rate of rise of the slow depolarization produced by depolarizing the neuronal membrane potential, whereas increasing the membrane potential elicited the opposite effect. Also, compare Figure $10 B$ with Figure $10 C$.

The regular pattern of discharge is corroborated by the unimodal distribution of action potentials observed when the oscilloscope sweeps were triggered by spontaneous spikes (Fig. 9C). The IPSPs locked to the cardiac cycle observed at normal values of arterial pressure were absent during hypotension, as demonstrated by the flat average of the neuronal membrane potentials triggered by the pulsatile arterial pressure (Fig. 9D).

As mentioned, during hyperpolarization the rate of rise of the slow depolarization decreases and, in consequence, the firing ratc is rcduced; nevertheless, the spikes remained unrelated to the pulse, as shown in Figure $10 E$. In this experimental condition, the hyperpolarization was achieved by constant current injection of $-0.3 \mathrm{nA}$. The average of the neuronal membrane potentials triggered by the pulsatile arterial pressure, performed during hypotension and hyperpolarization, was flat, even though a higher intensity of negative current was used $(-0.8 \mathrm{nA}$ ) (Fig. $10 F)$.

These results indicate that these RVLM bulbospinal neurons with barosensitive properties receive chloride-dependent IPSPs entrained to the cardiac cycle at normal values of arterial pressure. This modulation is no longer present during hypotension.

\section{Properties of barosensitive RVLM neurons with IPSPS}

The neurons described in Figures 6-10 with IPSPs locked to the pulsatile arterial pressure could be clearly differentiated from those ncurons recciving depolarizing potentials locked to the cardiac cycle described in Figures 1-4. In addition, the neurons inhibited by baroreceptor synaptic inputs were shown to have some characteristics revealed by hyperpolarizing and depolarizing pulses. The analysis of the voltage deflection elicited by the hyperpolarizing current pulse demonstrated a relatively fastdeveloping anomalous rectification that made the hyperpolarization decay $35-40 \%$ for deflections of the membrane potential of $15 \mathrm{mV}$ (Fig. $11 \mathrm{~A}$ ).

Depolarizing pulses in these neurons were associated with accommodation in the firing. The spikes induced by a depolar- 

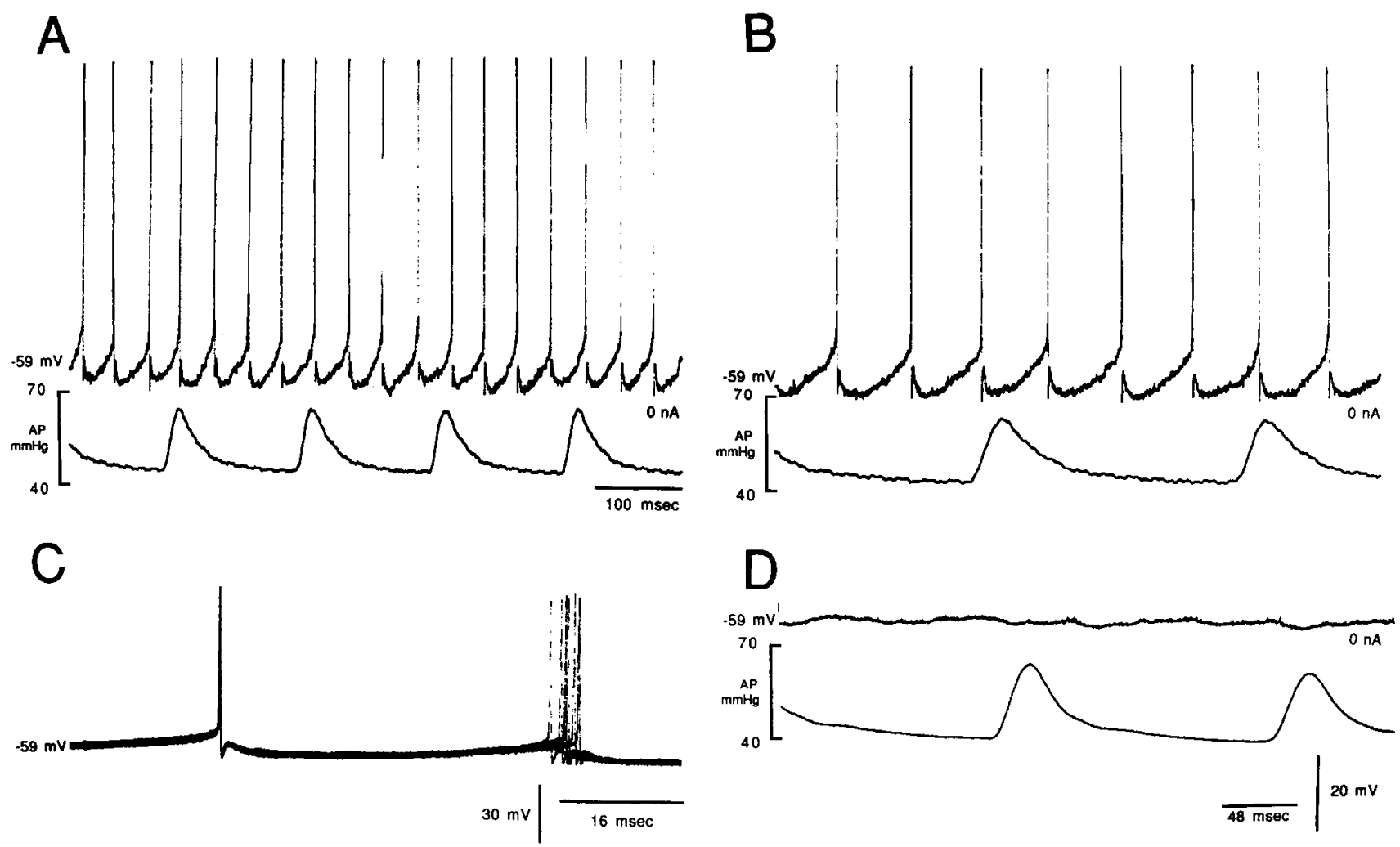

Figure 9. Intracellular recording of a RVLM bulbospinal neuron with inhibitory baroreceptor inputs during constant hypotension. $A$, Simultaneous records of arterial pressure $(A P)$ and intracellular recording at resting membrane potential during hypotension elicited by intravenous infusion of sodium nitroprusside. The records were triggered by the arterial pulse wave. Note the very regular firing pattern that took place when arterial pressure had been lowered. $B$, Similar records as in $A$, but at different time base. $C$, Seven superimposed oscilloscope traces triggered by spontaneous spikes. $D$, Simultaneous averages of the neuronal membrane potential and pulsatile arterial pressure triggered by the arterial pulse wave during hypotension. Number of sweeps, 690 ; sample period, $50 \mu \mathrm{sec}$. Experimental conditions in $A$ also applicable to $B-D$.

izing stimulus were followed by afterhyperpolarization (Fig. 11B). This mechanism of accommodation probably prevented these neurons from burst firing. In fact, under our experimental conditions, depolarizing pulses could not trigger a burst of action potentials in this type of neuron as, for instance, was observed in other types of RVLM neurons also modulated by baroreceptor inputs but without projections to the sympathetic spinal cord (not shown).

\section{Intracellular labeling of barosensitive RVLM bulbospinal neurons and double staining for PNMT immunoreactivity}

Intracellularly recorded and electrophysiologically characterized neurons in the RVLM were intracellularly injected with biocytin to define the location of these neurons in the medulla and to determine whether these labeled neurons demonstrated PNMT or TH immunoreactivity.

Twenty-five electrophysiologically characterized neurons with stable membrane potentials of more than $-46 \mathrm{mV}$ were well labeled and preserved. As illustrated in Figure 12, the intracellularly labeled neurons were in all cases located in the RVLM, in an area just caudal to the facial nerve nucleus that extended $0.7 \mathrm{~mm}$ in the rostrocaudalis coordinate: -3.0 to $-3.7 \mathrm{~mm}$ from the interaural line. However, the majority of these neurons were located in an area that corresponded with the rostral pole of the $\mathrm{Cl}$ adrenergic neuronal group (Granata et al., 1985), ventral to the nucleus ambiguus (retrofacial nucleus), and lateral to the inferior olive (Fig. 12C,B). The characterization of these bulbospinal neurons demonstrated that 14 of them were type
I, while 8 were characterized as type II. Four neurons were partially characterized either as type I or II (Fig. 12).

The histological sections containing the 21 labeled neurons and/or axodendritic processes were studied for their immunoreactivity to PNMT, while four labeled neurons underwent the TH immunocytochemical procedure, using in both cases the double labeling immunohistofluorescence procedure described in Materials and Methods. The results demonstrated that of the 21 neurons injected with biocytin and recovered, none demonstrated any PNMT immunoreactivity. Furthermore, not one of the four investigated neurons showed TH immunoreactivity. Special care was taken to analyze different dendritic processes for any trace of PNMT or TH immunoreactivity; nevertheless, the results were consistently negative. Figure $13, A$ and $C$, shows a barosensitive bulbospinal neuron in the RVLM, negative to PNMT immunoreactivity but surrounded by several PNMTimmunoreactive neurons (Fig. 13B,D). Figure 14, $A$ and $C$, illustrates another barosensitive neuron in the RVLM that did not demonstrate TH immunoreactivity, although it was found close to many catecholaminergic neurons (Fig. $14 B, D$ ). The proximity of this neuron to the ventral surface of the medulla is illustrated in Figure 15 A. Only 3 of 25 studied neurons were found to be separated by a distance of more than $320 \mu \mathrm{m}$ from the main catecholaminergic neuronal group. Figure $15 B$ illustrates a labeled neuron close to the ventral medullary surface in the external border of the RVLM, negative to PNMT immunoreactivity, and isolated from the cluster of $\mathrm{Cl}$ cells (not shown). 

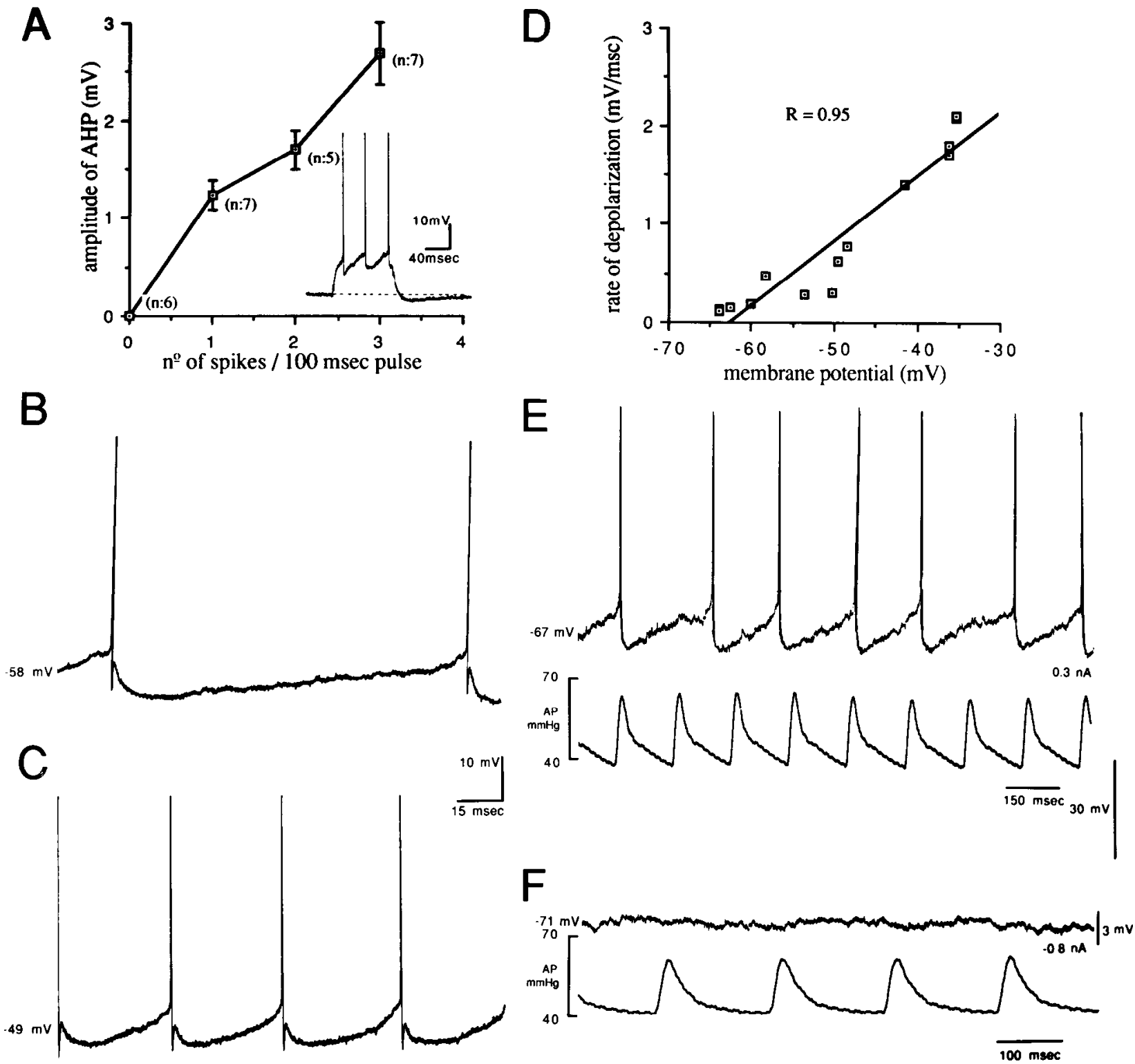

Figure 10. Influence of the number of spikes elicited in a depolarizing current pulse on the amplitude of the afterhyperpolarization ( $A H P$ ) spike train $(A)$. In $B-D$, effects of changes in neuronal membrane potential on the rate of rise of the slow depolarization in RVLM neurons are shown. In $E$ and $F$, effects of hyperpolarization and constant hypotension on a RVLM bulbospinal neuron with inhibitory baroreceptor inputs are shown. $A$, Intracellular injections of depolarizing current pulses $(100 \mathrm{msec})$ with different intensities elicited $0-3$ spikes in RVLM neurons. At the end of the current pulse, an afterhyperpolarization was observed, as illustrated in a typical case in $A$. The amplitude of this afterhyperpolarization was increased when the number of spikes evoked by the depolarizing pulses increased. $n$, number of cases. Values are means \pm SEM. $B-D$, During depolarization of the neuronal membrane potential, the rate of rise $(D, \mathrm{mV} / \mathrm{msec})$ of the slow depolarization increased; conversely, during hyperpolarization it decreased. The slope of the regression line was $70.0 \mu \mathrm{V} / \mathrm{msec} / \mathrm{mV}(n=14) . B$ and $C$ illustrate the slow depolarization at two different membrane potentials $(-58 \mathrm{mV}$ in $B-49 \mathrm{mV}$ in $C$ ). Note the same time base and calibration for $B$ and $C$. $E$, Simultaneous records of arterial pressure $(A P)$ and intracellular recording on the same neuron as in Figure 9 after intracellular injection of hyperpolarizing current for several minutes and intravenous infusion of sodium nitroprusside. The membrane potential was held at $-67 \mathrm{mV}$. The records were triggered by the arterial pulse wave. $F$, Simultaneous averages of the pulsatile arterial pressure with the neuronal membrane potential held at $-71 \mathrm{mV}$ during hypotension. The averages were triggered by the arterial pulse wave. Number of sweeps, 720 ; sample period, $100 \mu$ sec. Records $E$ and $F$ illustrate the same neuron.

\section{Discussion}

In this investigation, we used an intracellular recording technique in vivo to characterize bulbospinal neurons electrophysiologically in the RVLM with baroreceptor modulatory inputs. The recorded neurons were labeled by intracellular injections of biocytin. This procedure was combined with immunohistochemical localization of PNMT or TH, using a double labeling immunocytofluorescence technique (Kitai et al., 1989b; Kang and Kitai, 1990). In consequence, by using these methods, it was possible to determine whether functionally characterized neurons in the RVLM are themselves part of the $\mathrm{Cl}$ adrenergic group (Ross et al., 1984; Granata et al., 1985).

We found a population of spontaneously active neurons in the RVLM antidromically activated by stimulation of the IML in the thoracic spinal cord with axonal velocities of conduction ranging from 1.5 to $11.0 \mathrm{~m} / \mathrm{sec}$. These neurons were characterized because they were modulated by baroreceptor-related syn- 

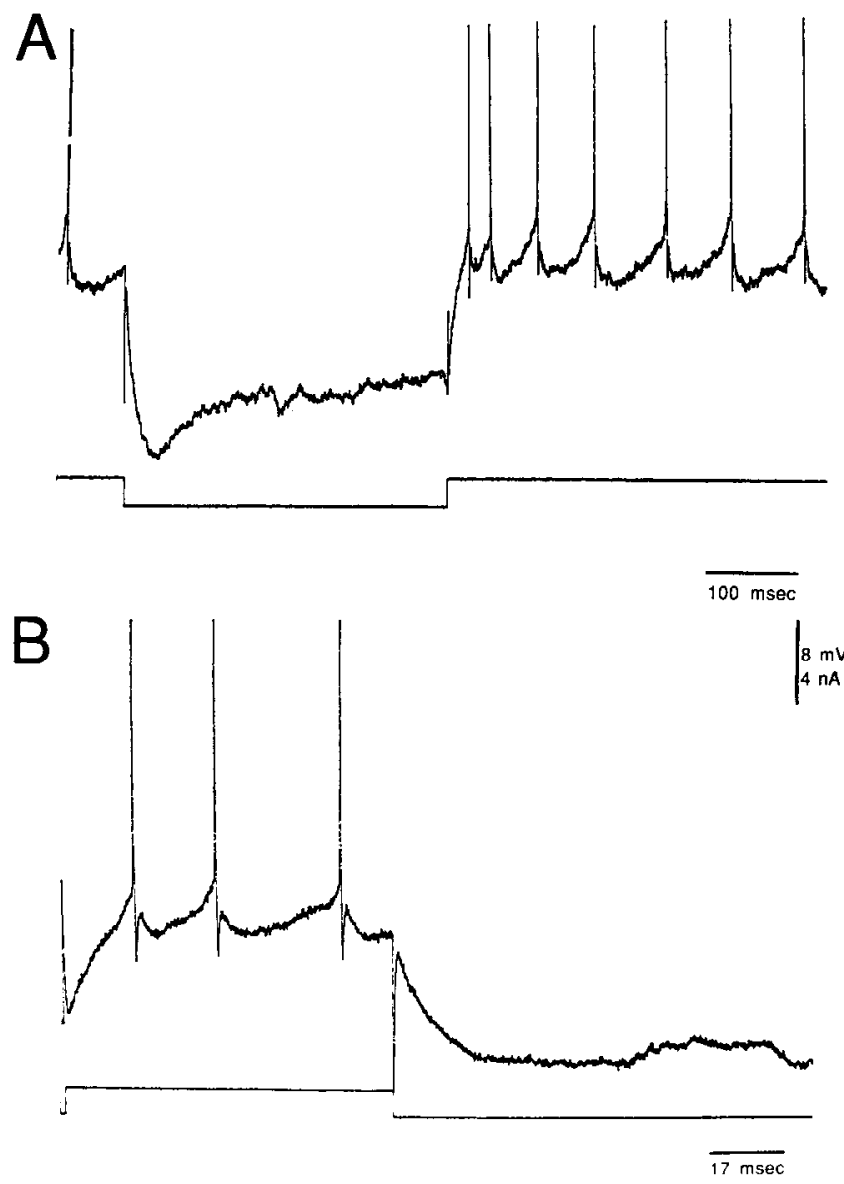

Figure 11. Responses of a physiologically identified RVLM bulbospinal neuron to intracellular current injections. $A$, At resting membrane potential $(-50 \mathrm{mV})$, a hyperpolarizing current pulse produced a twophase voltage deflection of the membrane potential. This time-dependent hang down in the voltage deflection trace is probably due to anomalous rectification. Note that after the offset of the current pulse, the frequency of discharge was transiently increased before the neuron recovered its regular firing pattern. $B$, Repetitive firing generated by a depolarizing current pulse. An initially higher frequency of activity was subsequently adapted to a lower level. At the termination of the pulse, an afterhyperpolarization was observed. These current injections were performed during a mean arterial pressure of $65 \mathrm{~mm} \mathrm{Hg}$ to avoid the interference of synchronized IPSPs. The intensity of the current injected is indicated beneath the traces.

aptic inputs. Furthermore, they were distinguished from a group of neurons located very close to the ventral medullary surface, also antidromically activated by electrical stimulation of the spinal cord with axonal conduction velocities ranging from 15.0 to $33.3 \mathrm{~m} / \mathrm{sec}$, because this second group of neurons did not show baroreceptor-modulated activity (Granata and Kitai, 1989). Nevertheless, some spontaneously active neurons in the RVLM also antidromically activated from the IML with similar axonal velocities of conduction (slower than $11 \mathrm{~m} / \mathrm{sec}$ ) did not dem-

Figure 12. Location of intracellularly recorded and labeled neurons $(A-C)$ : schematic representation of coronal sections of the medulla at $-3.7 \mathrm{~mm},-3.4 \mathrm{~mm}$, and $-3.0 \mathrm{~mm}$ from interaural line, respectively. The solid circles indicate the locations of the intracellularly labeled neurons electrophysiologically characterized as barosensitive type I, while the open circles indicate those neurons characterized as barosensitive
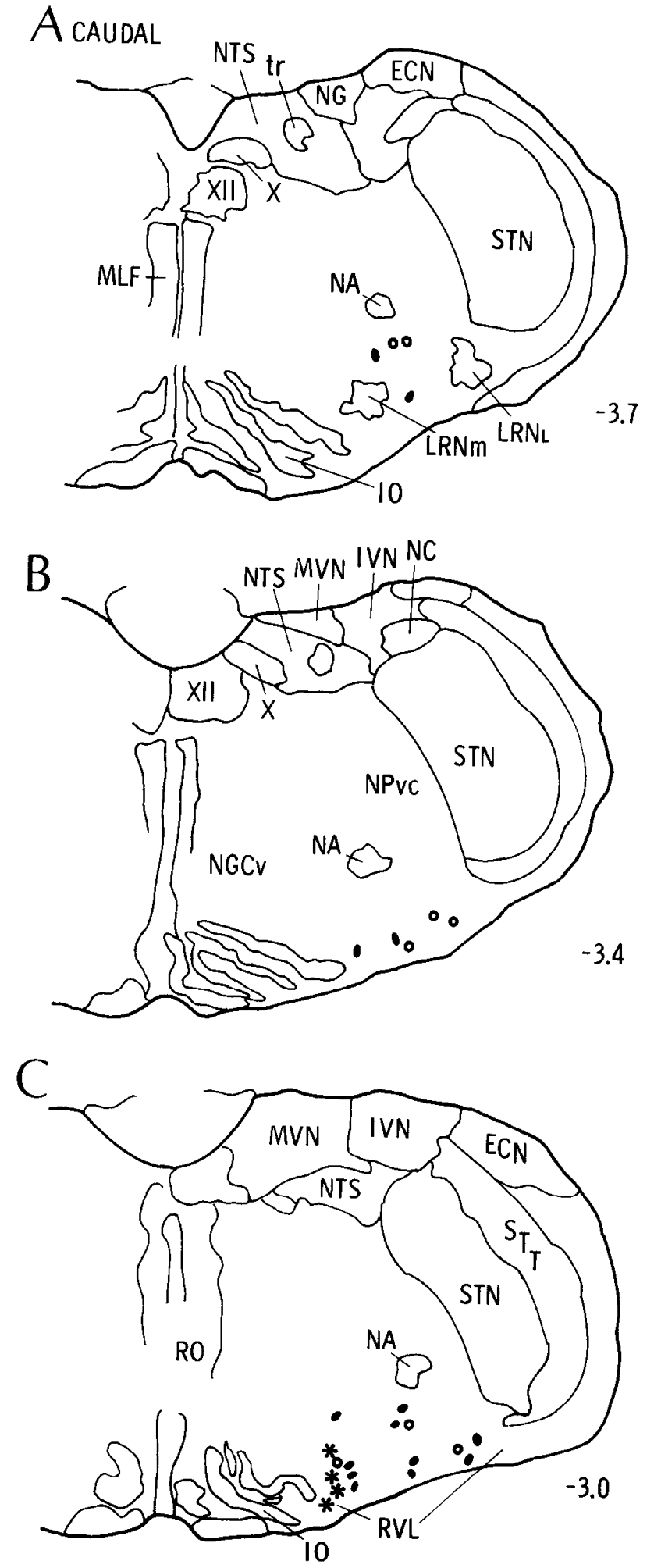

type II. The asterisks indicate the locations of partially characterized neurons. $E C N$, external cuneate nucleus; $I O$, inferior olive; $I V N$, inferior vestibular nucleus; $L R N L$, precerebellar lateral reticular nucleus lateralis; $L R N_{m}$, precerebellar lateral reticular nucleus medialis; $M L F$, mcdial longitudinal fasciculus; $M V N$, medial vestibular nucleus; $N A$, nucleus ambiguus; $N C$, nucleus cuneatus; $N G$, nucleus gracilis; $N G C v$, nucleus reticularis gigantocellularis (pars ventralis); $N P v c$, nucleus reticularis parvocellularis; NTS, nucleus tractus solitarius; $R O$, nucleus raphe obscurus; $R V L$, rostral ventrolateral medulla; $S T N$, spinal trigeminal nucleus; $S T T$, spinal trigeminal tract; $t r$, tractus solitarius; $X$, dorsal vagal motor nucleus; $X I I$, hypoglossal nucleus. 
onstrate pulse-related activity. On the contrary, they showed a very regular pattern of discharge.

The population of bulbospinal RVLM neurons with pulserelated activity consisted of at least two types: (1) RVLM-spinal neurons characterized by depolarizing potentials occurring at the end of the diastolic/beginning of the systolic pcriod. Thesc potentials increased in magnitude when the neuron was hyperpolarized by injections of negative current; the results then indicate that these potentials are probably EPSPs locked in the cardiac cycle. The baroreceptor reflex activation resulting from the increase in systemic arterial pressure evoked by phenylephrine elicited hyperpolarization with a decrease in the firing rate and an increase in the membrane input resistance. On the other hand, baroreceptor reflex inactivation produced depolarization with an increase in the frequency of discharge. Based on these characteristics, which can be distinguished from those of other neurons, we propose to classify these neurons as barosensitive type I. (2) RVLM-spinal neurons characterized by inhibitory potentials locked to the cardiac cycle at the end of the diastolic/ beginning of the systolic period. These potentials were found to be chloride-dependent IPSPs. In addition to the antidromic activation elicited by stimulation of the IML in the spinal cord, it was possible to evoke a shorter-latency monosynaptic EPSP in some of these neurons. Based on their electrophysiological characteristics, these neurons were classified as barosensitive type II. The phasic postsynaptic potentials received by both of these neurons at every cardiac cycle are likely to be the result of the rhythmic baroreceptor activation during each systole at normal values of arterial pressure. In support of this idea, the modulation of the membrane potential locked to the cardiac cycle was no longer present when the mean systemic arterial pressure was maintained below $70 \mathrm{~mm} \mathrm{Hg}$. These values of mean arterial pressure are below the activation threshold for arterial baroreceptor nerves (Chapleau and Abboud, 1987). During conditions of hypotension, the barosensitive neurons with pulse-synchronized IPSPs (characterized as type II) demonstrated (1) rhythmic and regular oscillations in membrane potential that probably control a constant interspike interval, and (2) an afterhyperpolarizing potential following the spike potential. These characteristics resembled a "beating" pacemaker-like activity. The speculative possibility that these neurons have pacemaker-like properties is based on (1) the regular pattern of spontaneous discharge revealed in the absence of baroreceptor inputs, (2) the absence of EPSPs when these neurons were hyperpolarized by negative current pulses up to below. spike threshold level, (3) the observation that interrupting the spontaneous activity by administering short pulses of hyperpolarizing current produced a delay in the spikes taking place after the current injection in relation to the expected time based on interspike interval before the current pulse [moreover, a single antidromic spike evoked on these neurons by spinal cord stimulation also produced a similar type of "resetting" of neuronal activity; however, when the antidromic spike collided with a spontaneous one, no change in the firing pattern was observed (A. R. Granata, unpublished observations)], and (4) the fact that the afterhyperpolarization potential and slow depolarizing potential present in these neurons are also observed in pacemaker neurons in other areas of the CNS (Grace, 1987). Bulbospinal neurons in the RVLM with paccmaker-like propertics have becn described by other investigators using extracellular recording in vivo and intracellular recording in vitro (Sun et al., 1988a,b). This pattern of activity, observed in these bulbospinal RVLM neurons in the absence of baroreceptor modulatory inputs due to hypotension, could be the origin of the ongoing synaptic activity observed in sympathetic preganglionic neurons, which originates at supraspinal levels. In fact, in spinal cats, a large proportion of sympathetic preganglionic neurons demonstrated very low levels of ongoing synaptic activity (Dembowsky et al., 1985). Furthermore, sympathetic background activity is almost completely suppressed after acute spinal cord transection (Alexander, 1946).

Another characteristic that distinguishes these type II neurons is the anomalous rectification developed by passing hyperpolarizing current pulses. This type of voltage-dependent conductance is present in ncurons with oscillatory bchavior, as, for instance, in the inferior olivary neurons (Yarom and Llinás, 1987) or substantia nigra neurons (Grace and Bunney, 1983; Bargas et al., 1988; Kubota et al., 1989), in which the level of anomalous rectification is directly related to the control of the frequency of the membrane oscillation.

Understanding the physiological role played by bulbospinal neurons characterized as type I will probably require additional data. However, their participation in the baroreceptor reflex is demonstrated by the depolarization with increases in the discharge rate evoked by the inactivation of arterial baroreceptors during systemic hypotension, as well as by the hyperpolarization accompanied by a reduction in the firing frequency and an increase in the input impedance following baroreceptor reflex activation. These results would indicate that the hyperpolarization elicited in these neurons by natural stimulation of arterial baroreceptors is caused by disfacilitation; this disfacilitation could result from the inhibition of tonic excitatory inputs generated in other neurons in the brainstem. These tonic excitatory inputs could be the origin of the important ongoing and random synaptic activity observed with these type I neurons at resting values of arterial pressure, heart rate, and neuronal membrane potential. Therefore, it is possible that the activation of arterial baroreceptors at a mean arterial pressure of $95 \mathrm{~mm} \mathrm{Hg}$ or more would inhibit these tonic excitatory neurons antecedent of the bulbospinal type I. However, at a mean arterial pressure below 95 $\mathrm{mm} \mathrm{Hg}$, these tonic excitatory neurons do not receive inhibitory baroreceptor inputs. On the other hand, the synchronized EPSPs locked to the cardiac cycle characterized under normotensive conditions cannot be explained by the aforementioned pathway. On the contrary, they may result from a polysynaptic excitatory baroreceptor pathway that is more conspicuous at a mean ar-

Figure 13. Photomicrograph of a bulbospinal neuron in the RVLM with pulse-modulated activity, intracellularly labeled with biocytin, and lacking PNMT immunoreactivity. Curved arrows in $A$ and $B$ indicate the ventral surface of the medulla. In $A$ and $C$, the neuron has been injected with biocytin and revealed by incubation of the tissue with avidin-Texas red. The neuron demonstrated intense fluorescence detected with a rhodamine filter. The tissue was subsequently processed for PNMT immunocytochemical localization, using FITC-labeled secondary antibodies. $B$ and $D$ show the same fields as in $A$ and $C$ but visualized through blue filter. Note that the neuron stained with biocytin (the two straight arrows indicate the localization of this neuron in $B$ and $D$ ) did not demonstrate PNMT immunoreactivity, although it is surrounded by several adrenergic neurons. Scale bar: $100 \mu \mathrm{m}$ for $A$ and $B, 25 \mu \mathrm{m}$ for $C$ and $D$. 


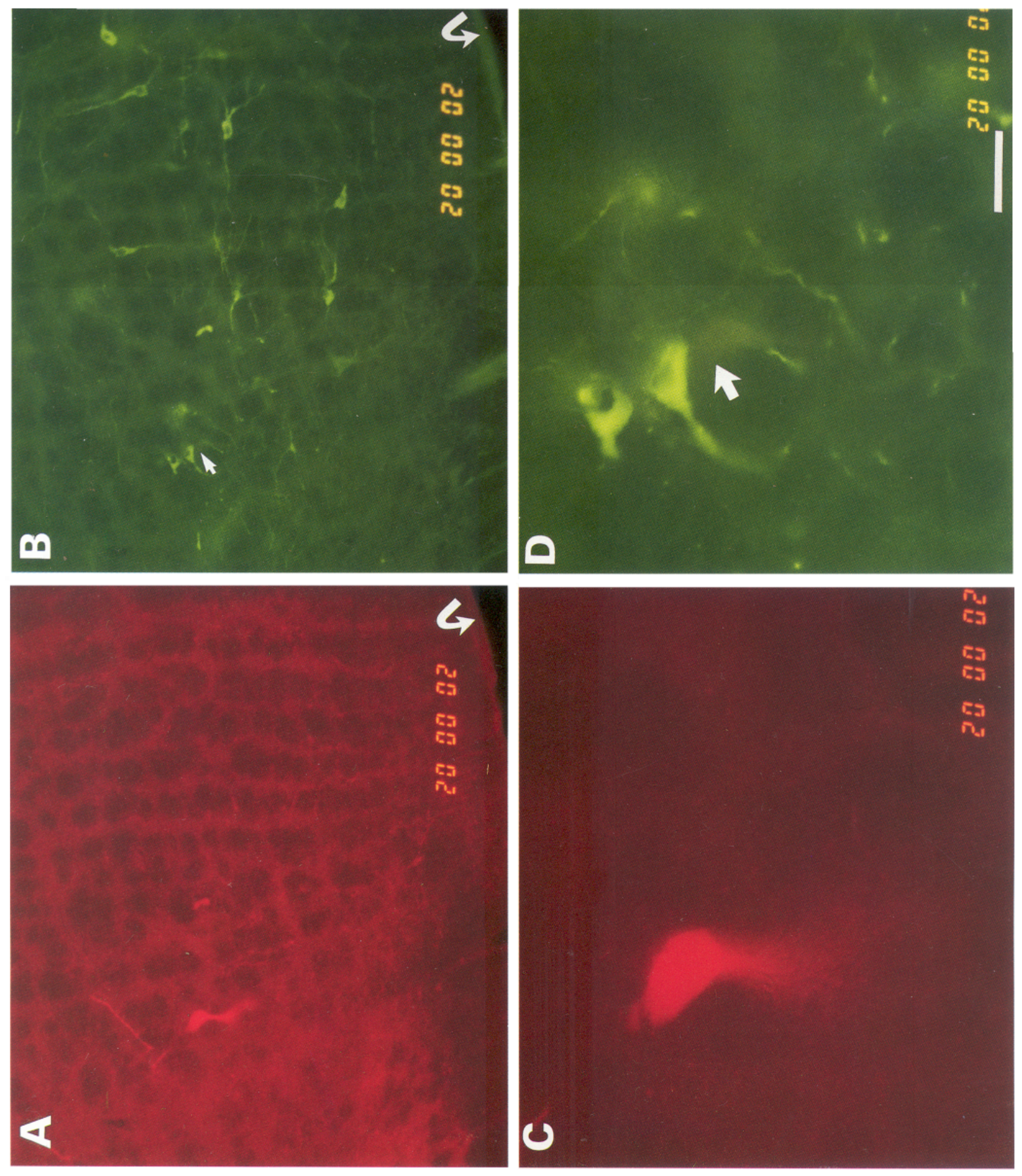



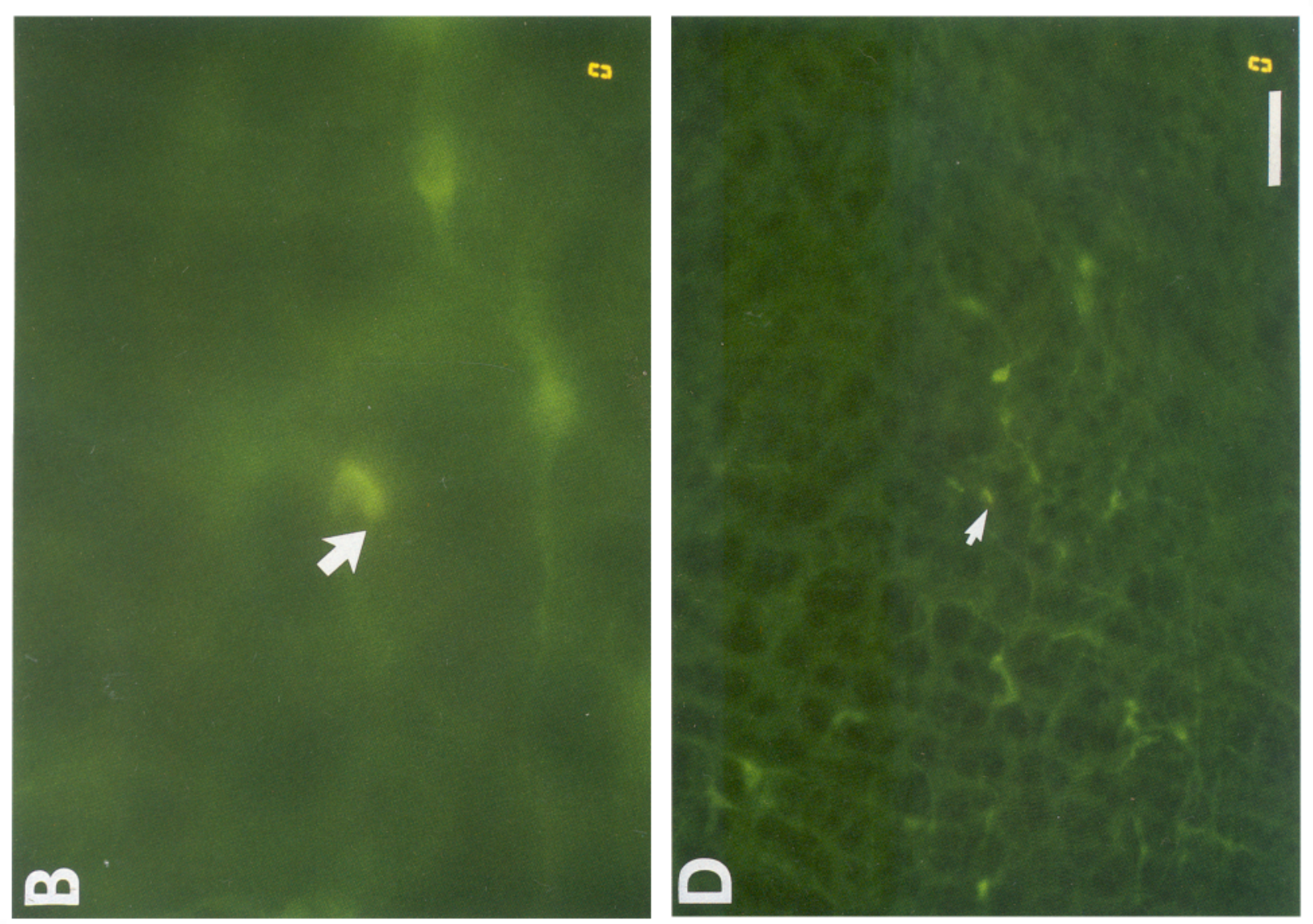

:

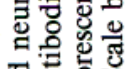

可

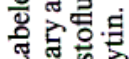

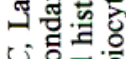

U

范要

헝

政

西学

응

윰도

100

年

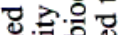

讨

बํํㄹ

$>0$

氜政

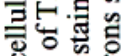

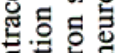

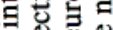

密.

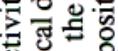

렬

诃

元

콘을

安品

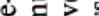

.

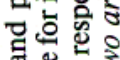

牙记

을

엉

물

․․․․

बु

के 은.

50.

至a

<记

政

娄 $0^{-\infty}$

ㄴ.

․ㅡㅁำ

등

드 땡밀

당현.

两运负

월 눙

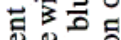

बू.

象

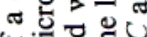

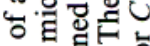

월

궁

o d

는. 퓽ㅇ

当嵒

의

인

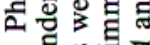

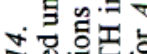

들

今 

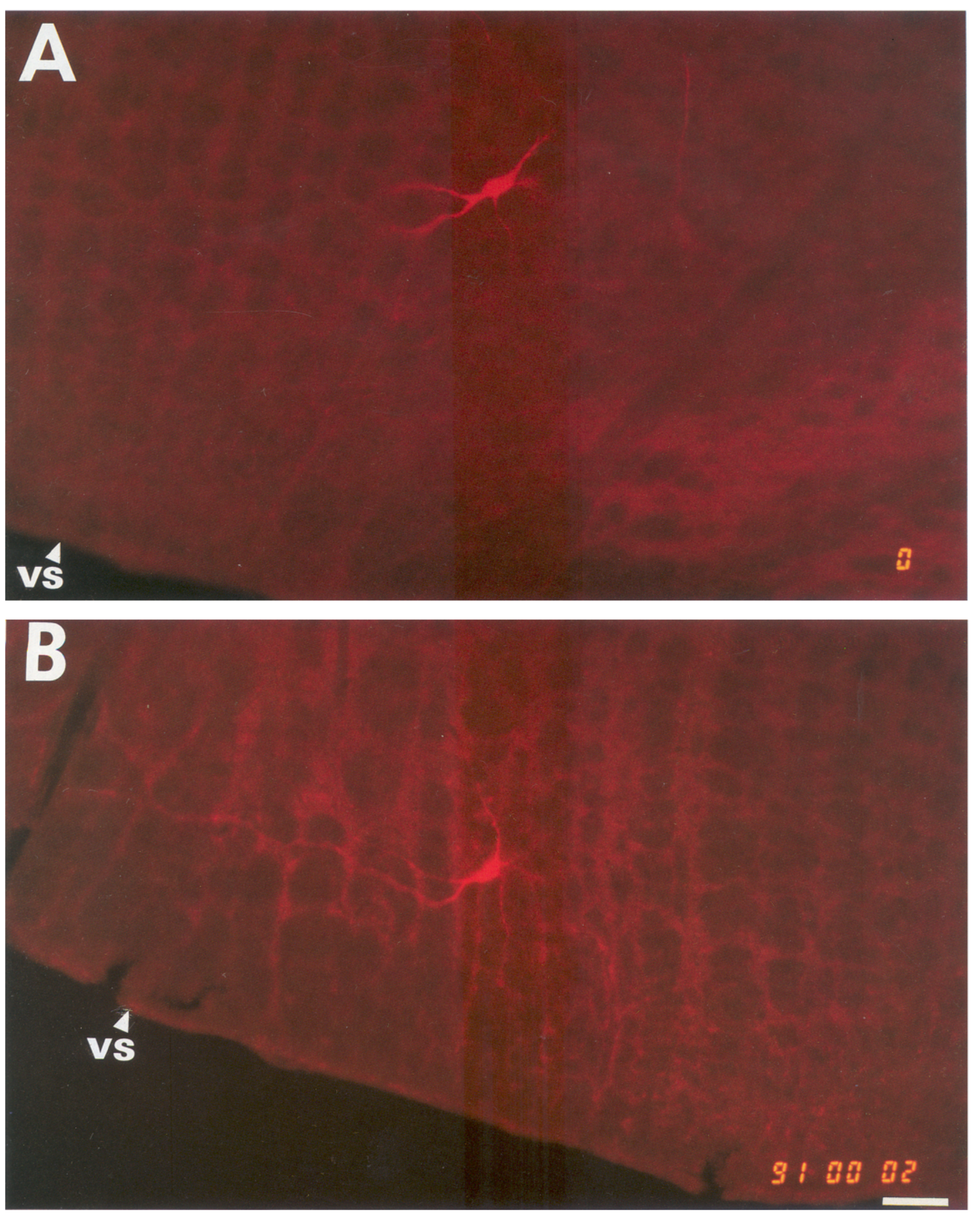

Figure 15. Photomicrograph of two physiologically characterized RVLM neurons, intracellularly labeled with biocytin. In $A$, the proximity to the ventral medullary surface $(V S)$ of the same neuron shown in Figure 14 is depicted in a different field. $B$, A different neuron intracellularly labeled with biocytin and revealed with avidin-Texas red histofluorescence. This neuron was located close to the ventral medullary surface in the external RVLM border and did not demonstrate PNMT immunoreactivity; neither was it located close to the main group of adrenergic neurons (not shown). Scale bar, $100 \mu \mathrm{m}$ for $A$ and $B$. 
terial pressure below $95 \mathrm{~mm} \mathrm{Hg}$ and probably plays some role in the entrainment of the sympathetic nerve activity to the cardiac cycle. The coupling of different neurons with synchronized EPSPs, such as the one described here, could play some role in the organization of sympathoexcitatory medullary circuits that discharge synchronous EPSPs to multiple groups of sympathetic preganglionic neurons in the IML, originating then the rhythmic component of the basal sympathetic nerve discharge. Therefore, it may well be that two different functional pathways, probably originating in second-baroreceptor-order neurons in the NTS, converge on barosensitive type I neurons. One of them would synchronize the neuronal activity at normal values of arterial pressure, while the second would shut the neuronal activity off when the systemic mean arterial pressure is increased above $95 \mathrm{~mm} \mathrm{Hg}$. In addition, at resting conditions, the spontaneous and random EPSPs received by type I neurons could drive the membrane potential to subthreshold level, allowing other synchronized baroreceptor excitatory inputs (EPSPs locked to the cardiac cycle) to take control of the neuronal activity.

The present results support the idea that the inhibition of the sympathetic vasomotor activity by arterial baroreceptor reflex inputs takes place at supraspinal levels, in particular in the RVLM (Granata et al., 1983, 1985; Yamada et al., 1984), and the neuronal groups characterized as barosensitive types I and II could participate in mediating these cardiovascular reflexes. Therefore, the sympathoinhibition mediated by baroreceptors could be, at least in part, the result of disfacilitation of sympathetic preganglionic neurons due to inhibition of these bulbospinal neurons by baroreceptor inputs. Although some reports claim that IPSPs are evoked in sympathetic preganglionic neurons by baroreceptor inputs (McLachlan and Hirst, 1980), we did not find RVLM neurons with IML projections that were excited by natural baroreceptor stimulation. Nearly $50 \%$ of the medullospinal neurons in the cat RVLM increased their firing rate upon stimulation of the arterial baroreceptor (Caverson et al., 1983), whereas in the rabbit this type of bulbospinal neuron could not be found (Terui et al., 1986). However, in rats less than $7 \%$ of medullospinal neurons with axonal velocities of conduction slower than $10 \mathrm{~m} / \mathrm{sec}$ were excited by baroreceptor activation (Brown and Guyenet, 1984). The sparsity of this neuronal population in the rat might explain the failure to characterize this type of neuron in the present report.

It has been reported that almost $20 \%$ of the RVLM neurons antidromically activated from the IML in rats had axonal conduction velocities slower than $1 \mathrm{~m} / \mathrm{sec}$, indicating that these neurons have unmyelinated axons (Morrison et al., 1988). Bulbospinal neurons with axonal conduction velocities less than 1 $\mathrm{m} / \mathrm{sec}$ were found also in rabbits (Terui et al., 1987), but not in cats (Barman and Gebber, 1985; McAllen, 1986). The present report gives no support for such slowly conducting RVLMspinal neurons with baroreceptor modulation. It is interesting to note that, according to Morrison et al. (1988), the majority of the RVLM-spinal neurons with nonmyelinated axons are silent. Therefore, the involvement of slowly conducting neurons in generating the tonic sympathoexcitatory drive to the IML could be insignificant. On the other hand, the spontaneously active bulbospinal neurons in the RVLM with axonal velocities of conduction varying from 1.5 to $11.0 \mathrm{~m} / \mathrm{sec}$ described in this study could participate in generating and mediating the tonic sympathoexcitatory drive to the IML. In support of this idea, we found that single square pulses of cathodal current applied to similar areas of the RVLM evoked a composite excitatory response on the renal sympathetic nerve (RSN) as the result of stimulation of sympathoexcitatory descending pathways (Granata, unpublished observations). The subtraction of the conduction time of the RSN response evoked by IML stimulation (at $\mathrm{T}_{2}-\mathrm{T}_{3}$ level) from the RVLM-RSN conduction time demonstrates conduction velocities for the bulbospinal component of the RVLM sympathoexcitatory response similar to the antidromic velocities of conduction of RVLM-spinal barosensitive neurons described in this report (Granata, unpublished observations).

The electrical stimulation of the IML in the spinal cord, in addition to antidromic potentials, also evoked monosynaptic EPSPs with a shorter onset latency in barosensitive type II neurons. This distinctive characteristic could be the result of activating spinomedullary projections, probably the afferent pathway mediating the somatic pressor reflex (Stornetta et al., 1989) or the stimulation of spinothalamic branching axons on RVLM neurons. On the other hand, it is also possible that these monosynaptic EPSPs produced by spinal cord stimulation were evoked by stimulation of recurrent collaterals to the RVLM originating from descending projections terminating in or passing through the IML. These monosynaptic EPSPs could play some role in synchronizing the activity of several sympathoexcitatory bulbospinal neurons. In any case, the fact these EPSPs could follow a very high frequency of stimulation (near $1000 \mathrm{~Hz}$ ) demonstrates this is a very safe excitatory synapse.

Neurons with rhythmic activity in phase with the spontaneous ventilation did not demonstrate the typical one-to-one relationship of neuronal rhythmic activity and pulsatile arterial pressure observed in barosensitive neurons. However, from the present analysis, we cannot totally exclude the possibility that a different type of correspondence could exist between the cardiac and neuronal rhythms. Still, when the membrane potentials of these neurons were examined over a long time frame, no clear rhythmic fluctuations entrained to the arterial pressure pulse were observed. Other investigators have reported that respiratory neurons do not receive baroreceptor modulatory inputs (Richter and Seller, 1975).

One of the aims of this study was to test the hypothesis that neurons of the adrenergic group in the RVLM (C1 neurons) are essential for mediating vasodepressor responses from arterial baroreceptors, as well as for generating cardiovascular sympathetic nerve activity. The present results demonstrate that two different populations of bulbospinal RVLM neurons exist. Both groups are barosensitive, and their spontaneous neuronal activity is modulated by baroreceptor inputs. Neither group produces PNMT or has TH immunoreactivity. These results give strong support to the view that noncatecholaminergic neurons in the RVLM with spinal projections are involved in the tonic and reflex control of cardiovascular parameters.

The double labeling technique used to investigate whether the intracellularly injected neurons display a catecholaminergic phenotype has been used successfully in other catecholaminergic neurons in the CNS intracellularly injected with biocytin. Those experiments showed that the biocytin-avidin-Texas red histofluorescence reaction does not interfere with immunohistofluorescence staining with catecholaminergic or other antibodies (Bargas et al., 1988; Kang and Kitai, 1990).

The intracellularly recorded neurons characterized as barosensitive were located in the RVLM in an area defined in previous publications as the $\mathrm{Cl}$ area (Ross et al., 1983, 1984; 
Granata et al., 1985, 1986), which includes the bulk of PNMTcontaining neurons, as well as a group of adrenergic neurons situated more medially in the rostral pole of the RVLM. The majority of the labeled neurons were either close to or intermingled with the PNMT-containing neurons. However, few of them were found separated from the $\mathrm{C} 1$ group in an area close to the ventrolateral border of the RVLM. Some intracellularly labeled neurons in the RVLM were located very close to the $\mathrm{Cl}$ neurons, in two cases overlapping each other. Therefore, to better distinguish the injected neurons from surrounding ones, the fluorescent FITC image was converted to permanent DAB staining (see Materials and Methods). Under these procedures, it was clear that the two intracellularly injected neurons were not catecholaminergic. In consequence, we were persuaded to conclude that the population of cardiovascular neurons investigated in this study is more likely not catecholaminergic. Nevertheless, from these results, we cannot exclude the possibility that $\mathrm{Cl}$ adrenergic neurons are themselves involved in cardiovascular regulation as well. It is possible to speculate that (1) PNMT-containing neurons are also spontaneously active and barosensitive, but the present experimental conditions are biased against recording from these cells, or (2) the $\mathrm{C} 1$ group could belong to the slowly conducting nonmyelinated RVLM-spinal neurons, which, according to Morrison et al. (1988), are mostly silent. Therefore, if that is the case, the role played by $\mathrm{C} 1$ neurons in cardiovascular control is doubtful, at least under our experimental conditions. (3) Finally, the participation of these adrenergic neurons in association with other descending neurons using different transmitters in mediating either the well-established vasomotor function or some other refiexes (somato- and viscerosympathetic reflexes, chemosensory function, etc.) is still unknown.

The pacemaker-like pattern of discharge observed in barosensitive type II neurons during hypotension is quite similar to that found in other neurons in the brain with positive immunoreaction to catecholaminergic biosynthetic enzymes, such as, for instance, the dopaminergic neurons in the substantia nigra pars compacta (Kitai et al., 1989a) and locus ceruleus noradrenergic neurons (Williams et al., 1984). In spite of that, noncatecholaminergic neurons in the CNS also show a pacemakerlike pattern of discharge, such as, for instance, neurons in the pedunculopontine area (Granata and Kitai, 1991).

In summary, the results presented in this study provide direct evidence for two different types of barosensitive bulbospinal neurons in the RVLM. Barosensitive type I neurons, with excitatory potentials synchronized to the cardiac cycle and a disfacilitatory mechanism, account for hyperpolarization during constant baroreceptor activation above $95 \mathrm{~mm} \mathrm{Hg}$ of mean arterial pressure. Barosensitive type II neurons, with $\mathrm{Cl}$-dependent IPSPs locked to the cardiac cycle and likely produced by baroreceptor inputs, and with a pacemaker-like basic pattern of discharge, are revealed when baroreceptor inputs are abolished by hypotension. Neither type I nor type II barosensitive neurons demonstrated PNMT immunoreactivity; therefore, these results support the idea that noncatecholaminergic medullospinal neurons in the RVLM also may play an important role in the tonic and reflex control of cardiovascular function.

\section{References}

Alexander RS (1946) Tonic and reflex functions of medullary sympathetic cardiovascular centers. J Neurophysiol 9:205-217.

Amendt K, Czachurski J, Dembowsky K, Seller H (1979) Bulbospinal projections to the intermediolateral cell column: a neuroanatomical study. J Auton Nerv Syst 1:103-117.

Bargas J, Galarraga E, Chang HT, Kitai ST (1988) Electrophysiological and double-labelling immunohistochemical analysis of neurons in the substantia nigra zona compacta in the rat. Soc Neurosci Abstr 14: 1025.

Barman SM, Gebber GL (1985) Axonal projection patterns of ventrolateral medullospinal sympathoexcitatory neurons. J Neurophysiol 53:1551-1566.

Benarroch EE, Granata AR, Ruggiero DA, Park DH, Reis DJ (1986) Neurons of $\mathrm{Cl}$ area mediate cardiovascular responses initiated from ventral medullary surface. Am J Physiol 250:R932-R945.

Brown DL, Guyenet PG (1984) Cardiovascular neurons of brain stem with projections to spinal cord. Am J Physiol 247:R1009-R1016.

Caverson MM, Ciriello J, Calaresu FR (1983) Direct pathway from cardiovascular neurons in the ventrolateral medulla to the region of the intermediolateral nucleus of the upper thoracic cord: an anatomical and electrophysiological investigation in the cat. $J$ Auton Nerv Syst 9:451-475.

Chapleau MW, Abboud FM (1987) Contrasting effects of static and pulsatile pressure on carotid baroreceptor activity in dogs. Circ Res 61:648-658.

Coote JH (1988) The organization of cardiovascular neurons in the spinal cord. Rev Physiol Biochem Pharmacol 110:147-185.

Coote JH, MacLeod VH, Fleetwood-Walker S, Gilbey MP (1981) The response of individual sympathetic preganglionic neurons to microelectrophoretically applied endogenous monoamines. Brain Res 215 : 135-145.

Dampney RAL, Moon EA (1980) Role of ventrolateral medulla in vasomotor response to cerebral ischemia. Am J Physiol 239:H349H358.

Dembowsky K, Czachurski J, Seller H (1985) An intracellular study of the synaptic input to sympathetic preganglionic neurones of the third thoracic segment of the cat. J Auton Nerv Syst 13:201-244.

Grace AA (1987) The regulation of dopamine neuron activity as determined by in vivo and in vitro intracellular recordings. In: Neurophysiology of dopaminergic systems-current status and clinical perspectives (Chiodo LA, Freeman AS, eds), pp 1-66. Grosse Pointe, MI: Lakeshore Press.

Grace AA, Bunney BS (1983) Intracellular and extracellular electrophysiology of nigral dopaminergic neurons. I. Identification and characterization. Neuroscience 10:301-315.

Granata AR, Kitai ST (1989) Intracellular analysis in vivo of cardiovascular neurons in the rostral ventrolateral medulla. Soc Neurosci Abstr 15:812.

Granata AR, Kitai ST (1990) Characterization of different cardiovascular neurons in the rostral ventrolateral medulla. An in vivo intracellular study. FASEB J 4:A1192.

Granata AR, Kitai ST (1991) Inhibitory substantia nigra inputs to the pedunculopontine neurons. Exp Brain Res, in press.

Granata AR, Ruggiero DA, Park DH, Joh TH, Reis DJ (1983) Lesions of epinephrine neurons in the rostral ventrolateral medulla abolish the vasodepressor components of baroreflex and cardiopulmonary reflex. Hypertension 5:V80-V84.

Granata AR, Ruggiero DA, Park DII, Joh TH, Reis DJ (1985) Brainstem area with $\mathrm{Cl}$ epinephrine neurons mediates baroreflex vasodepressor responses. Am J Physiol 248:H547-H567.

Granata AR, Numao Y, Kumada M, Reis DJ (1986) Al noradrenergic neurons tonically inhibit sympathoexcitatory neurons of $\mathrm{Cl}$ area in rat brainstem. Brain Res 377:127-146.

Hilton SM, Marshall JM, Timms RJ (1983) Ventral medullary relay neurones in the pathway from the defence areas of the cat and their effect on blood pressure. J Physiol (Lond) 345:149-166.

Hökfelt T, Fuxe K, Goldstein M, Johansson O (1974) Immunohistochemical evidence for the existence of adrenaline neurons in rat brain. Brain Res 66:235-251.

Horikawa K, Armstrong WE (1988) A versatile means of intracellular labeling: injection of biocytin and its detection with avidin conjugates. J Neurosci Methods 25:1-11.

Howard BR, Tabatabai M (1975) Localization of the medullary respiratory neurons in rats by microelectrode recording. J Appl Physiol 39:812-817.

Joh TH, Goldstein M (1973) Isolation and characterization of multiple forms of phenylethanolamine $N$-methyltransferase. Mol Pharmacol 9:117-129. 
Kang YN, Kitai ST (1990) Electrophysiological propertics of pedunculopontine neurons and their postsynaptic responses following stimulation of substantia nigra reticulata. Brain Res 535:79-95.

Kitai ST, Kang YN, Kubota Y (1989a) The regulation of rhythmic firing of substantia nigra compacta dopaminergic neurons. Soc Neurosci Abstr 15:901.

Kitai ST, Penny GR, Chang H (1989b) Intracellular labeling and immunocytochemistry. In: Neuroanatomical-tract tracing methods 2 (Heimer L, Zaborszky L, eds), pp 173-199. New York: Plenum.

Kubota Y, Kang YN, Kitai ST (1989) Two types of substantia nigra compacta neurons based on electrophysiological membrane characteristics. Soc Neurosci Abstr 15:900.

Lipski J (1981) Antidromic activation of neurons as an analytic tool in the study of the central nervous system. J Neurosci Methods 4:132.

Loewy AD, Burton $\mathrm{H}$ (1978) Nuclei of the solitary tract: efferent projections to the lower brainstem and spinal cord of the cat. J Comp Neurol 181:421-450.

Loewy AD, McKellar S (1981) Serotonergic projections from the ventral medulla to the intermediolateral cell column in the rat. Brain Res 211:146-152.

Lovick TA (1985) Projections from the diencephalon and mesencephalon to nucleus paragigantocellularis lateralis in the cat. Neuroscience 14:853-861.

Lovick TA (1988) Convergent afferent inputs to neurones in nucleus paragigantocellularis lateralis in the cat. Brain Res 456:183-187.

McAllen RM (1985) Mediation of the fastigial pressor response and a somatosympathetic reflex by ventral medullary neurones in the cat. J Physiol (Lond) 368:423-433.

McAllen RM (1986) Identification and properties of sub-retrofacial bulbospinal neurones: a descending cardiovascular pathway in the cat. J Auton Nerv Syst 17:151-164.

McAllen RM, Neil JJ, Loewy AD (1982) Effects of kainic acid applied to the ventral surface of the medulla oblongata on vasomotor tone, the baroreceptor reflex and hypothalamic autonomic responses. Brain Res 238:65-76.

McLachlan EM, Hirst GDS (1980) Some properties of preganglionic neurons in upper thoracic spinal cord of the cat. J Neurophysiol 43: 1251-1265.

Milner TA, Morrison SF, Abate C, Reis DJ (1988) Phenylethanolamine $N$-methyltransferase containing terminals synapse directly on sympathetic preganglionic neurons in the rat. Brain Res 448:205222.

Morrison SF, Milner TA, Reis DJ (1988) Reticulospinal vasomotor neurons of the rat rostral ventrolateral medulla: relationship to sympathetic nerve activity and the $\mathrm{Cl}$ adrenergic cell group. J Neurosci 8:1286-1301.

Richter DW, Seller H (1975) Baroreceptor effects on medullary respiratory neurones of the cat. Brain Res 86:168-171.

Ross CA, Ruggiero DA, Joh TH, Park DH, Reis DJ (1983) Adrenaline synthesizing neurons in the rostral ventrolateral medulla: a possible role in tonic vasomotor control. Brain Res 273:356-361.
Ross CA, Ruggiero DA, Park DH, Joh TH, Sved AF, Fernandcz-Pardal J, Saavedra JM, Reis DJ (1984) Tonic vasomotor control by the rostral ventrolateral medulla: effect of electrical or chemical stimulation of the area containing $\mathrm{C} 1$ adrenaline neurons on arterial pressure, heart rate and plasma catecholamines and vasopressin. J Neurosci 4:474-494.

Ross CA, Ruggiero DA, Reis DJ (1985) Projections from the nucleus tractus solitarii to the rostral ventrolateral medulla. J Comp Neurol 242:511-534.

Ruggiero DA, Reis DJ (1988) Neurons containing phenylethanolamine $N$-methyltransferase: a component of the baroreceptor reflex? In Epinephrine in the central nervous system (Stolk JM, U'Prichard DC, Fuxe K, eds), pp 291-307. New York: Oxford UP.

Sternberger LA (1979) Immunocytochemistry. New York: Wiley.

Stornetta R, Morrison SF, Ruggiero DA, Reis DJ (1989) Neurons in the rostral ventrolateral medulla mediate the somatic pressor reflex. Am J Physiol 256:R448-R462.

Sun MK, Guyenet PG (1985) GABA-mediated baroreceptor inhibition of reticulospinal neurons. Am J Physiol 249:R672-R680.

Sun MK, Guyenet PG (1986) Hypothalamic glutamatergic input to medullary sympathoexcitatory neurons in rats. Am J Physiol 251: R798-R810.

Sun MK, Hackett JT, Guyenet PG (1988a) Sympathoexcitatory neurons of rostral ventrolateral medulla exhibit pacemaker properties in the presence of a glutamate-receptor antagonist. Brain Res 438:2340.

Sun MK, Young BS, Hackett JT, Guyenet PG (1988b) Reticulospinal pacemaker neurons of the rat rostral ventrolateral medulla with putative sympathoexcilatory function: an intracellular study in vitro. Brain Res 442:229-239.

Terui N, Saeki Y, Kumada M (1986) Barosensory neurons in the ventrolateral medulla in rabbits and their responses to various afferent inputs from peripheral and central sources. Jpn J Physiol 36:11411164.

Tucker DC, Saper CB, Ruggiero DA, Reis DJ (1987) Organization of central adrenergic pathways. I. Relationships of ventrolateral medullary projections to the hypothalamus and spinal cord. J Comp Neurol 259:591-603.

Williams JT, North RA, Shefner SA, Nishi S, Egan TM (1984) Membrane properties of rat locus ceruleus neurons. Neuroscience 13:137156.

Yamada KA, McAllen RM, Loewy AD (1984) GABA antagonists applied to the ventral surface of the medulla oblongata block the baroreceptor reflex. Brain Res 297:175-180.

Yarom Y, Llinás R (1987) Long-term modifiability of anomalous and delayed rectification in guinea pig inferior olivary neurons. J Neurosci 7:1166-1177.

Yoshimura M, Polosa C, Nishi S (1989) Multiple actions of noradrenaline on sympathetic preganglionic neurons of the cat studied in the spinal cord slice. Prog Brain Res 81:181-190. 